\title{
APLICACIÓN DE LA TÉCNICA H/V EN DISTINTOS EMPLAZAMIENTOS A PARTIR DE LA MEDICIÓN DE VIBRACIONES AMBIENTALES Y DE SISMOS
}

\author{
APPLICATION OF H/V TECHNIQUE ON DIFFERENT SOILS USING \\ AMBIENT VIBRATION MEASUREMENTS AND EARTHQUAKES
}

\author{
Víctor Schmidt-Díaz \\ Laboratorio de Ingeniería Sísmica. Nivel 3-A Facultad de Ingeniería, \\ Universidad de Costa Rica, 2060, San Pedro de Montes de Oca \\ victor.schmidt@ucr.ac.cr
}

(Recibido: 19/05/2015; aceptado: 9/11/2015)

\begin{abstract}
Four techniques to identify the fundamental frequency (f0) for 26 different sites were applied. These techniques correspond to spectral ratios based on the recording of: ambient vibration using an accelerograph (denominated VAA), ambient vibration using a seismograph (VAS), total seismic records (ST) and a time window of $5 \mathrm{~s}$ staring at $\mathrm{S}$ waves arrives in the seismic records (SOS). Better results were achieved when VAA was applied during the day (at 8 am and $3 \mathrm{pm}$ ). When VAA at $3 \mathrm{pm}$ was compared to VAS at day time, it was observed that in the $58 \%$ of the analyzed cases the shapes and amplitudes of the spectral ratios were different, which means that VAA is not an appropriated technique to identify f0 based on that the results from VAS are the correct ones. It is affirmed based on its similarity to ST and SOS techniques in a $75 \%$ of the studied cases. These last two techniques are better justified in both mathematical and physical fundaments.

Keywords: Spectral ratio, ambiet vibration, earthquakes, fundaental period, soils.

RESUMEN: Se aplicaron cuatro técnicas para la identificación de la frecuencia fundamental para 26 distintos emplazamientos. Estas corresponden a las razones espectrales obtenidas a partir del registro de: vibraciones ambientales con el uso de un acelerógrafo (VAA), vibraciones ambientales utilizando un sismógrafo (VAS), sismos completos (ST) y ventanas de 5 segundos a partir del arribo de las ondas "S" (SOS). En la aplicación de VAA, se lograron mejores resultados cuando la prueba se aplicó en horas del día ( 8 am y 3 pm). Al comparar VAA a las 3 pm con VAS en horas del día, en un 58\% de los casos analizados se observan formas y amplitudes distintas, lo que descalifica el uso de VAA para la identificación de f0 ya que se toma como el resultado correcto el proporcionado por VAS, tal y como lo proponen diversos autores y en esta investigación en específico se evidenció su semejanza a las técnicas ST y SOS, en un 75\% de los casos. Estas dos últimas técnicas están mejor justificadas según su fundamento físico y matemático.

Palabras clave: Razón espectral, vibraciones ambientales, sismos, periodo fundamental, suelos.
\end{abstract}




\section{INTRODUCCIÓN}

Uno de los parámetros dinámicos más importantes utilizados en la ingeniería sísmica es el período fundamental $\mathrm{T}_{0}$ de un emplazamiento (o su inverso que es la frecuencia $f_{0}$ ). Si se visualiza al suelo como un filtro, las ondas que atraviesen ese medio se verán amplificadas en las frecuencias que muestren mayor amplitud en la función de transferencia y es precisamente en $\mathrm{f}_{0}$ donde se produce la mayor amplificación de todas.

$\mathrm{Si}$ además esta $\mathrm{f}_{0}$ coincide con la frecuencia fundamental de las estructuras ubicadas sobre el emplazamiento en estudio, se produce el fenómeno de resonancia al generarse un sismo, lo que puede traer como consecuencia efectos destructivos importantes en las obras civiles, como desplazamientos significativos entre pisos sucesivos, generación de rótulas plásticas cerca de las uniones viga-columna, daños significativos en elementos no estructurales, etc.

Es por estas razones que una adecuada identificación de $\mathrm{f}_{0}$ es muy importante, más aún porque esta puede modificarse en el tiempo. Por ejemplo, si la sacudida es muy intensa (sismo fuerte) el suelo tendrá un comportamiento no lineal, lo que usualmente implica una disminución de $\mathrm{f}_{0}$ (incremento de $\mathrm{T}_{0}$ ). También, los procesos de consolidación natural o artificial del suelo, pueden generar modificaciones en el tiempo de este parámetro.

Existen diversos métodos con los que puede estimarse $\mathrm{f}_{0} \mathrm{y}$ su amplificación., los cuales pueden clasificarse como: directos, empíricos y numéricos:

-Directos: razón espectral estándar tradicional, que determina la amplificación en un sitio específico respecto a otro de referencia. La respuesta de sitio se obtiene simplemente con la división del espectro de amplitudes de Fourier calculado a partir del registro obtenido en la estación en estudio, con respecto al espectro del acelerograma del mismo sismo y misma dirección pero obtenido en el sitio de referencia, que debe estar libre de efectos de sitio (amplificación igual en todo el rango de frecuencias cercando a la unidad). Normalmente se utiliza como sitio de referencia aquel donde existe afloramiento de la roca (Reipl et al., 1998). Esta es una técnica difícil de aplicar en Costa Rica, ya que es muy poco frecuente encontrar tales condiciones en los sitios más densamente poblados, que corresponden a valles y llanuras donde predominan suelos de origen aluvial, lacustre o depósitos de ceniza volcánica.

-Empíricos: funciones de recibimiento (receiver function) o $\mathrm{H} / \mathrm{V}$ : amplificación obtenida usando la razón espectral de la componente horizontal respecto a la vertical para un mismo sismo. No se requiere de un sitio de referencia, por lo que es muy conveniente su uso cuando la roca no aflora y más bien si existen grandes extensiones de sedimentos. La componente vertical es asumida como libre de la influencia de la superficie. Se utiliza solamente un instrumento triaxial, por lo que resulta ser una técnica muy fácil de aplicar. Sus bases teóricas no son fáciles de justificar, por lo que se considera un método empírico (Reipl et al., 1998).

Esta técnica también se puede aplicar para ventanas de tiempo de corta duración, entre 5 y 10 $\mathrm{s}$ inmediatamente después del arribo de las ondas "S", que es la zona del registro denominada como "onda de coda". Su forma espectral se supone que es independiente de la posición del instrumento de medición, así como de la localización de la fuente, debido a que esta parte de la señal está predominantemente influenciada por las ondas reflejadas, debido a heterogeneidades en la corteza.

Esta técnica puede aplicarse también en forma semejante, pero a partir de registros de ruido o vibraciones ambientales, tradicionalmente conocida como técnica de Nakamura (Nakamura, 1989), que será descrita en profundidad en el siguiente apartado.

-Numéricos: a partir de un perfil de suelos debidamente detallado (cada capa caracterizada por su espesor, velocidad de onda cortante (Vs), amortiguamiento $(\xi)$ y densidad) se crea la función de transferencia del medio, de manera que se logre identificar la frecuencia de máxima amplificación, que corresponde a $\mathrm{f}_{0}$.

Debido a que el suelo puede mostrar un comportamiento no lineal de acuerdo con el nivel de deformación inducida que está estrechamente relacionada con la sacudida a que es sometido el medio, existen algoritmos que permiten generar esta función de transferencia a partir de un sismo 
de entrada (input colocado en la base del perfil). Un ejemplo de un método numérico simplificado que sigue esta metodología es el programa Shake (Schnabel et al., 1972), denominado lineal equivalente ya que en forma iterativa, compatibiliza los niveles de deformación en cada capa y estima los parámetros dinámicos asociados. Para cada iteración se calcula una deformación efectiva y se estiman Vs (o el módulo de rigidez G) y $\xi$ asociados según curvas existentes, seleccionadas de acuerdo con el tipo de suelo. A partir de estos valores, se resuelve esa iteración específica como si fuera un caso lineal. Se comparan los valores de deformación efectiva entre dos iteraciones sucesivas hasta lograr que sean muy parecidas. Así logra crear la función de transferencia entre dos puntos cualesquiera del perfil de suelo.

De los métodos brevemente descritos en párrafos anteriores, en esta investigación se tratarán los definidos como empíricos, a partir de distintas condiciones de aplicación.

\section{MARCO TEÓRICO}

\section{Técnica $\mathrm{H} / \mathrm{V}$ aplicada en términos generales}

La idea de obtener la razón espectral entre las componentes horizontal y vertical $(\mathrm{H} / \mathrm{V})$ registradas en un solo instrumento (un único sitio de medición) fue primeramente introducida por Nogoshi \& Igarashi (1971) quienes mostraron la relación entre esa razón $\mathrm{H} / \mathrm{V}$ con la curva de elipticidad de las ondas Rayleigh e identificaron la coincidencia entre el máximo de la más baja frecuencia observada en $\mathrm{H} / \mathrm{V}$ con la frecuencia fundamental de resonancia, para usarla como indicador de la estructura del suelo debajo del sitio de registro (Bard, 1999).

Diversos autores como Lachet \& Bard (1994), Konno \& Ohmachi (1998) coinciden en lo siguiente:

La razón $\mathrm{H} / \mathrm{V}$ está básicamente relacionada con la elipticidad de las ondas Rayleigh, debido a la predominancia de ondas Rayleigh en la componente vertical.

Esta elipticidad es dependiente de la frecuencia y muestra un pico definido cerca de la frecuencia fundamental en sitios donde existe un contraste entre impedancias significativo (superior a la unidad), dado por la siguiente relación (Kramer, 1996):

$$
\alpha=(\rho 2 v 2) /(\rho 1 v 1)
$$

Donde: $\alpha$ representa la razón de impedancias, $v_{1}, v_{2}$ las velocidades de onda cortante de las capas 1 (en superficie) y 2 (en profundidad) y $\rho_{1}, \rho_{2}$ las densidades para los mismos materiales 1 y 2 .

En general, se considera que un contraste entre impedancias $\alpha$ superior a 3, permite la identificación de un pico sobresaliente, que corresponde a la frecuencia predominante del sitio bajo estudio (Nogoshi \& Igarashi, 1971).

\section{Técnica $\mathrm{H} / \mathrm{V}$ aplicada a partir de vibraciones ambientales}

Propuesto por Nakamura (1989), este método ha probado ser útil para la identificación del periodo predominante de depósitos de suelo, siempre y cuando existan estudios previos de la geología local, así como sondeos geotécnicos y geofísicos que permitan aplicar otras técnicas en forma paralela, de manera que los resultados puedan ser comparados y contrastados, debido a que la técnica no siempre produce resultados confiables.

Como se dijo antes, consiste en la determinación de los espectros de Fourier de las componentes horizontales y de la vertical de registros de vibraciones ambientales, obtenidos con un velocímetro o un acelerógrafo triaxial. A partir de ellos se obtiene la razón H/V (cociente espectral), que es considerado por el autor como la función de transferencia aproximada de los estratos del suelo sobre el basamento. Al contarse con dos componentes horizontales, $\mathrm{H}$ suele ser considerado como el promedio de los espectros en el plano horizontal, ya sea la media aritmética o la media geométrica.

Las vibraciones ambientales (o ruido como lo denominan algunos autores) tienen dos orígenes: natural y humano. El cuadro 1 muestra la diferenciación entre estos dos tipos y que representa el consenso al que llegó el grupo del proyecto SESAME (2004) quien se ha dedicado durante varios años al estudio y uso de este tipo de mediciones. 
Desde el punto de vista de su justificación, el método $\mathrm{H} / \mathrm{V}$ parte de que las vibraciones ambientales están compuestas por varios tipos de ondas y considera que estas son similares horizontal y verticalmente en el basamento. Las vibraciones son amplificadas por las distintas capas del suelo, sufriendo las componentes horizontales multirreflexiones de la onda S, por lo que se ven amplificadas mientras que la componente vertical sufre un fenómeno semejante pero para la onda P. La figura 1 muestra la terminología que se emplea en la deducción del método.

La función de transferencia de los estratos superficiales es:

$$
\mathrm{ST}=\mathrm{SHS} / \mathrm{SHB}
$$

Nakamura (1989) asume que el movimiento vertical no se ve amplificado por las capas superficiales. Propone además el siguiente término:

$$
\mathrm{ES}=\mathrm{SVS} / \mathrm{SVB}
$$

Que representa el efecto de la onda Rayleigh en el movimiento vertical. Si no hay ondas Rayleigh, ES = 1; si aumenta el efecto de estas ondas, ES se vuelve mayor que uno.

El cociente $\mathrm{STT}=\mathrm{ST} / \mathrm{ES}$ es considerado como una función de transferencia más confiable, ya que se ha logrado eliminar el efecto de las ondas Rayleigh. Entonces:

$$
\mathrm{STT}=\mathrm{ST} / \mathrm{ES}=(\mathrm{SHS} / \mathrm{SHB}) /(\mathrm{SVS} / \mathrm{SVB})=
$$
$(\mathrm{SHS} * \mathrm{SVB}) /(\mathrm{SHB} * \mathrm{SVS})=(\mathrm{SHS} / \mathrm{SVS}) /(\mathrm{SHB} /$ $\mathrm{SVB})=\mathrm{RS} / \mathrm{RB}$

Donde:

RS representa la división de los espectros $\mathrm{H}$ y $\mathrm{V}$ en superficie (S).

$\mathrm{RB}$ representa la división de los espectros $\mathrm{H}$ y V en el basamento (B).

$\mathrm{RB}$ es aproximadamente igual a la unidad para un rango amplio de frecuencias, cosa que se puede probar a partir de registros obtenidos en el basamento.

Siendo así, STT $=$ RS $=$ SHS/SVS representa la función de transferencia buscada. Esto significa que el movimiento vertical en superficie contiene las características del movimiento horizontal en el basamento.

Nakamura (1989) basa su teoría en las siguientes premisas:

1- Se tienen en cuenta los efectos de las ondas Rayleigh.

2- Los efectos de las ondas Rayleigh son iguales en la componente horizontal y en la vertical. De ahí se establece STT.

\begin{tabular}{|c|c|c|}
\hline Origen de las vibraciones & Natural & Humano \\
\hline Nombre & Microsismos & Microtremores (microtemblores) \\
\hline $\begin{array}{c}\text { Frecuencia que predomina en la } \\
\text { vibración (aprox.) }\end{array}$ & $0.1 \mathrm{~Hz}$ a $1 \mathrm{~Hz}$ (baja frecuencia) & $1 \mathrm{~Hz}$ a $10 \mathrm{~Hz}$ (alta frecuencia) \\
\hline Fuente & $\begin{array}{l}\text { Oceánico y condiciones atmosféricas } \\
\text { (frecuencia muy baja) }\end{array}$ & Tráfico, industria \\
\hline Onda incidente & Ondas superficiales (fuentes lejanas) & $\begin{array}{l}\text { Ondas superficiales }+ \text { ondas internas (fuente } \\
\text { cercana) }\end{array}$ \\
\hline Variación en amplitud & Relacionada con ondas oceánicas & Día/noche, semana/fin de semana \\
\hline Rayleigh/Love & Predominante Rayleigh & $\begin{array}{l}\text { Amplitud comparable, Love con un poco } \\
\text { más de energía }\end{array}$ \\
\hline $\begin{array}{l}\text { Modo fundamental/ } \\
\text { modos superiores }\end{array}$ & Principalmente fundamental & $\begin{array}{l}\text { Posibilidad de modos más altos en frecuen- } \\
\text { cias altas (al menos en el caso de dos capas) }\end{array}$ \\
\hline
\end{tabular}

Cuadro 1

Diferenciación de las vibraciones ambientales según SESAME (2004) 


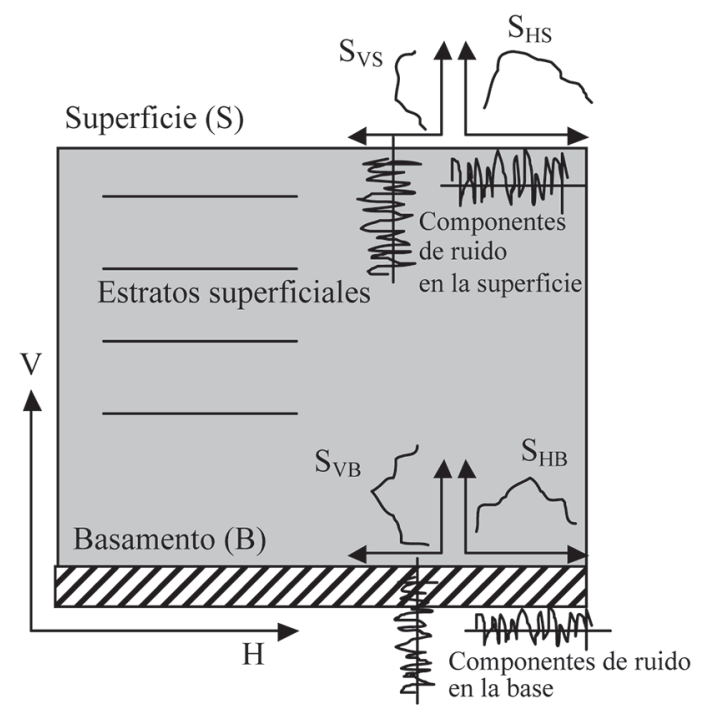

Fig.1: Esquema a partir del cual se deduce la técnica de Nakamura.

3- Como SHB/SVB es aproximadamente igual a la unidad, STT se obtiene de la razón espectral entre la componente horizontal y la vertical.

Varios autores sostienen que el método está basado en suposiciones débiles y que requiere comprobaciones de campo. Por ejemplo, Kudo (1995) plantea que la segunda premisa indicada en la figura 1 no es válida porque la composición de las vibraciones no puede ser expresada como una convolución de ondas internas y superficiales, sino que son generalmente una suma de estos dos tipos de ondas; entonces ST/ES no genera ninguna compensación de ondas Rayleigh.

La tercera suposición es únicamente válida si en las vibraciones ambientales predominan ondas Rayleigh y su elipticidad es casi vertical, pero generalmente esto sucede en medios estratificados donde hay un contraste significativo entre las impedancias del medio estratificado y del basamento, por lo tanto, es una hipótesis no siempre válida.

En resumen, a pesar de la ausencia de justificaciones teóricas convincentes, la técnica funciona de manera experimental y su uso es muy extendido, por lo que se justifica su estudio en profundidad y desde distintas ópticas.

\section{METODOLOGÍA}

En el cuadro 2 se muestra los sitios donde se realizaron las pruebas, específicamente el nombre de la estación acelerográfica, las coordenadas, y los tipos de mediciones hechas. Se señala además los casos en que se aplicó alguna de las técnicas $($ con $\sqrt{ }$ ) o si no se hizo (X).

El tipo de medición realizado en cada sitio se detalla seguidamente:

VAA, vibraciones ambientales registradas con acelerógrafo: corresponden a registros de aceleración obtenidos por instrumentos triaxiales marca Reftek, de 24 bits, modelo SMA con memoria de estado sólido (MEMS) y una escala completa de $2 \mathrm{~g}$. La figura 2 muestra este instrumento y la figura $2 \mathrm{~b}$ se muestra la forma en que se ancla al suelo para evitar que, ante la ocurrencia de un sismo fuerte, se produzca una amplificación del movimiento debido al movimiento relativo entre el acelerógrafo y el suelo. Las mediciones fueron hechas en tres distintas horas del día: $2 \mathrm{am}$, 8 am y 3 pm, para comparar las razones espectrales según el nivel de ruido presente. En todos los casos, las ventanas de tiempo utilizadas fueron de 15 minutos.

VAS, vibraciones ambientales registradas con sismógrafo: se utilizó un instrumento triaxial marca Lennartz, modelo LE-3D/20s conectado a un digitalizador Reftek de seis canales, tal y como lo muestra la figura 3. Las mediciones se realizaron cerca del acelerógrafo instalado en forma permanente, durante horas del día. En todos los casos se registraron ventanas de 15 minutos de duración. En la fig. 3b se observa que el instrumento no se ancla al sitio, sino simplemente se apoya en superficie, sea esta una losa o el suelo. Esto es válido ya que la técnica VAS solamente se aplicó para la medición de vibraciones ambientales, que son de muy baja intensidad y que no va a generarse una posible amplificación debida al movimiento relativo del sensor respecto a la superficie.

$\mathrm{ST}$, sismos registrados con acelerógrafos, registro total: se revisaron, uno a uno, todos los registros acelerográficos obtenidos en el LIS desde 2009 hasta 2014, eliminándose los que mostraban formas de onda incompletas o con una relación 
Cuadro 2

Coordenadas de los sitios y pruebas aplicadas

\begin{tabular}{|c|c|c|c|c|c|c|c|}
\hline Nombre de sitio & Estación & Latitud & Longitud & VAA & VAS & ST & SOS \\
\hline Sede INS, Alajuela & AALA & 10,025 & $-84,2137$ & $\sqrt{ }$ & $\sqrt{ }$ & $\sqrt{ }$ & $\sqrt{ }$ \\
\hline Clínica Hatillo & SHTH & 9,91474 & $-84,0967$ & $\sqrt{ }$ & $\sqrt{ }$ & $\sqrt{ }$ & $\sqrt{ }$ \\
\hline Cruz Roja de Palmares & APMR & 10,0574 & $-84,4357$ & $\sqrt{ }$ & $\sqrt{ }$ & $\sqrt{ }$ & $\sqrt{ }$ \\
\hline Sede UCR, Paraiso & CPAR & 9,82727 & $-83,8687$ & $\sqrt{ }$ & $\sqrt{ }$ & $\sqrt{ }$ & $\sqrt{ }$ \\
\hline Estación de Bomberos, Quepos & PQUE & 9,43107 & $-84,1638$ & $\sqrt{ }$ & $\mathrm{X}$ & $\sqrt{ }$ & $\sqrt{ }$ \\
\hline Sede UCR, Rodrigo Facio & SLPF & 9,936 & $-84,052$ & $\sqrt{ }$ & $\sqrt{ }$ & $\sqrt{ }$ & $\sqrt{ }$ \\
\hline ICODER (documentos) & SSBN & 9,93473 & $-84,0994$ & $\sqrt{ }$ & $\sqrt{ }$ & $\sqrt{ }$ & $\sqrt{ }$ \\
\hline Sede UCR, Occidente & ASRM & 10,0867 & $-84,4784$ & $\sqrt{ }$ & $\sqrt{ }$ & $\sqrt{ }$ & $\sqrt{ }$ \\
\hline Biblioteca Municipal, Cartago & CCRT & 9,86424 & $-83,9271$ & $\sqrt{ }$ & $\sqrt{ }$ & $\sqrt{ }$ & $\sqrt{ }$ \\
\hline Instituto Tecnológico de Costa Rica & CTEC & 9,85425 & $-83,9074$ & $\sqrt{ }$ & $\sqrt{ }$ & $\sqrt{ }$ & $\sqrt{ }$ \\
\hline Sede UCR, Fabio Baudrit & AFBR & 10,0072 & $-84,26565$ & $\sqrt{ }$ & $\sqrt{ }$ & $\sqrt{ }$ & $\sqrt{ }$ \\
\hline Sede UCR, Tacares & AGRE & 10,0419 & $-84,2998$ & $\sqrt{ }$ & $\sqrt{ }$ & $\sqrt{ }$ & $\sqrt{ }$ \\
\hline Cruz Roja, Jacó & PJAC & 9,6094 & $-84,6228$ & $\sqrt{ }$ & $\mathrm{X}$ & $\sqrt{ }$ & $\sqrt{ }$ \\
\hline Municipalidad de Puerto Cortés & POSA & 8,9795 & $-83,5321$ & $\sqrt{ }$ & $\mathrm{X}$ & $\sqrt{ }$ & $\sqrt{ }$ \\
\hline Saint Jude School & SJUD & 9,95 & $-84,203$ & $\sqrt{ }$ & $X$ & $\sqrt{ }$ & $\sqrt{ }$ \\
\hline Sede UCR, Turrialba & CTBA & 9,903 & $-83,671$ & $\sqrt{ }$ & $\mathrm{X}$ & $\sqrt{ }$ & $\sqrt{ }$ \\
\hline Municipalidad de Sarapiquí & HPV J & 10,4498 & $-84,0133$ & $\sqrt{ }$ & $\mathrm{X}$ & $\sqrt{ }$ & $X$ \\
\hline Cruz Roja la Virgen & HVRG & 10,3906 & $-84,1394$ & $\sqrt{ }$ & $\mathrm{X}$ & $\sqrt{ }$ & $\mathrm{X}$ \\
\hline Biblioteca Municipal de Ciudad Quesada & ASCS & 10,324 & $-84,431$ & $\sqrt{ }$ & $\mathrm{X}$ & $\sqrt{ }$ & $\mathrm{X}$ \\
\hline San Miguel & HCPD & 9,99512 & $-84,0358$ & $\sqrt{ }$ & $\mathrm{X}$ & $\sqrt{ }$ & $\mathrm{X}$ \\
\hline Cruz Roja, Orotina & AORT & 9,91213 & $-84,5222$ & $\sqrt{ }$ & $\mathrm{X}$ & $\sqrt{ }$ & $\mathrm{X}$ \\
\hline Sede UCR, Liberia & GLIB & 10,618044 & $-85,45815$ & $\sqrt{ }$ & $\mathrm{X}$ & $\sqrt{ }$ & $\mathrm{X}$ \\
\hline Sede INS, Nicoya & GNYA & 10,145 & $-85,4527$ & $\sqrt{ }$ & $\mathrm{X}$ & $\sqrt{ }$ & $\mathrm{X}$ \\
\hline Cruz Roja de Heredia & HHDA & 10,0002 & $-84,1168$ & $\sqrt{ }$ & $\mathrm{X}$ & $\sqrt{ }$ & $\mathrm{X}$ \\
\hline Sede UCR, Guápiles & LGPI & 10,213 & $-83,771$ & $\sqrt{ }$ & $\mathrm{X}$ & $\sqrt{ }$ & $\mathrm{X}$ \\
\hline Sede del INS en Siquirres & LSQR & 10,0958 & $-83,5046$ & $\sqrt{ }$ & $X$ & $\sqrt{ }$ & $\mathrm{X}$ \\
\hline Sede UCR, Fraijanes & AFRA & 10,13707 & $-84,1931$ & $\sqrt{ }$ & $\mathrm{X}$ & $\sqrt{ }$ & $\mathrm{X}$ \\
\hline
\end{tabular}

señal ruido inadecuada (inferior a 3 , según la técnica STA/LTA, Duval et al., 2001). El final del registro se consideró aproximadamente igual al tiempo en el que el nivel de ruido antes y después del sismo fuera el mismo. En términos generales, los acelerogramas que cumplieron con estas características provienen de sismos con $\mathrm{Mw}$ $\geq 4,0$. Para aplicar esta técnica, es necesario el registro de sismos con acelerógrafos ya que son instrumentos diseñados para medir el movimiento fuerte del suelo, por lo tanto las amplitudes de las trazas no se ven saturadas, como si sucede con la mayoría de sismógrafos. Esta técnica también es conocida como HVSR por sus siglas en inglés. Para estas mediciones se usaron acelerógrafos como los descritos para la técnica VAA.

SOS, sismos registrados con acelerógrafos, registro de onda $\mathrm{S}$ : a partir de una inspección visual de cada registro, se tomó una ventana de 5 segundos para aplicar esta técnica (Rielp et al., 1998). Para estas mediciones se usaron acelerógrafos como los descritos para la técnica VAA.

Para lograr obtener la razón espectral de la componente horizontal sobre la vertical, en todos 

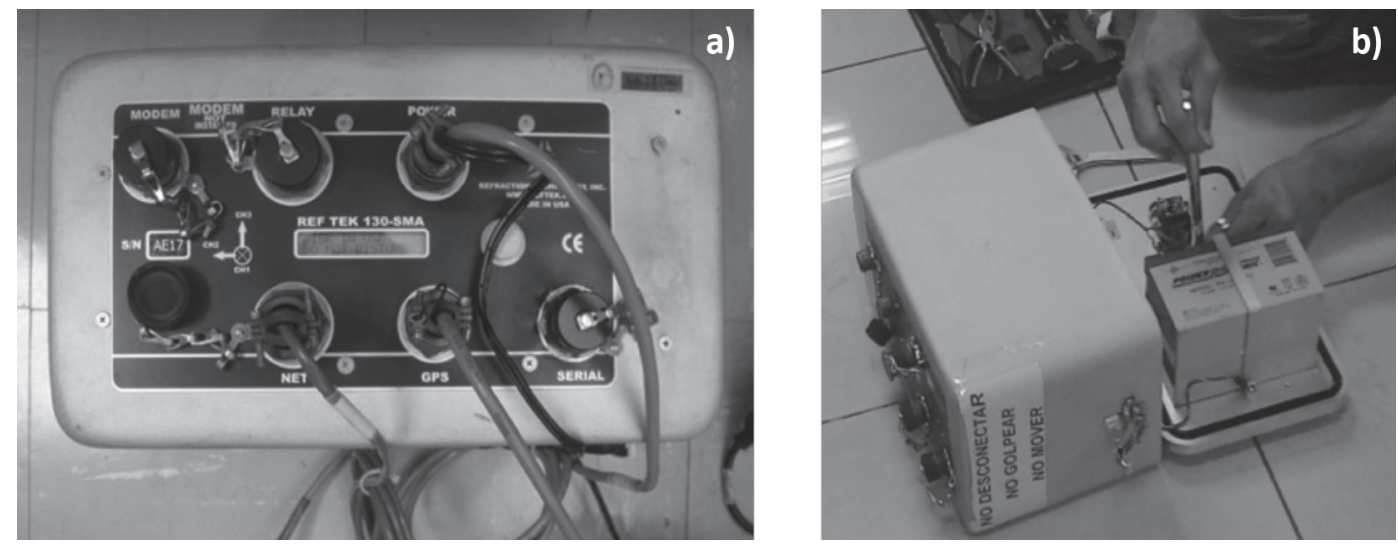

Fig. 2: Acelerógrafo marca Reftek, modelo SMA (2a). Sujeción al suelo por medio de un perno (2b).

los casos anteriores se aplicó la transformada rápida de Fourier (FFT) a las dos componentes horizontales y a la vertical por cada registro y de esta manera se obtuvo los espectros de amplitudes.

En el caso de VAA y VAS, el procesamiento se llevó a cabo con el programa GEOPSY versión 2.9.0 (SESAME European Group, www. geopsy.org). De acuerdo con la metodología seguida por este algoritmo, para cada frecuencia se calculó la media geométrica de las dos amplitudes horizontales y este valor se dividió entre la amplitud de la vertical.

Para determinar el tamaño de cada ventana temporal a partir de la cual se calculó el espectro, se consideró que debían estar presentes al menos 30 periodos según la frecuencia que se estuviera considerando. En vista de que se generan varias razones espectrales para cada frecuencia (mayor cantidad para frecuencias altas), GEOPSY obtiene un promedio de estas razones, que es el que se utilizó en esta investigación.

Para ST y SOS, se trabajó con una única ventana de tiempo que corresponde a la duración total de cada registro y para las dos amplitudes horizontales por frecuencia se calculó la media cuadrática.

Como puede verse en el cuadro 2 , de los 26 sitios bajo estudio, los tipos de medición VAA y ST
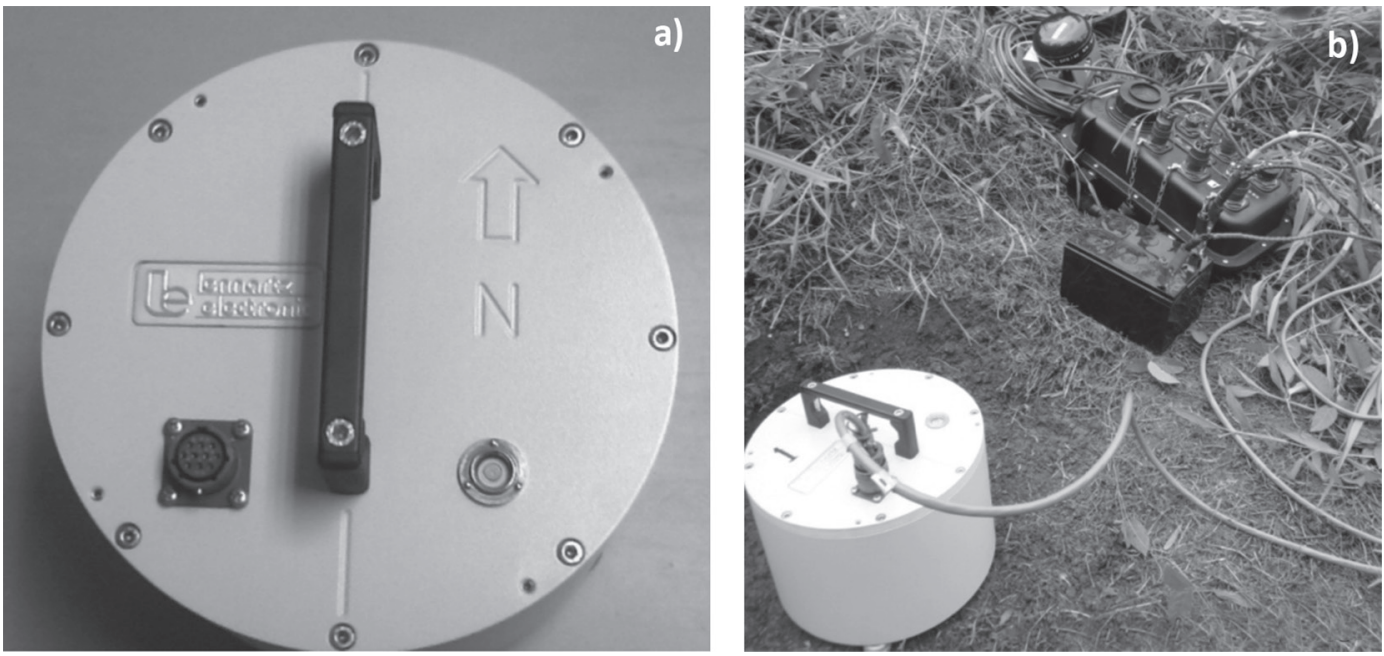

Fig. 3: Sismógrafo marca Lennartz (3a), modelo LE-3D/20s conectado a un digitalizado Reftek de seis canales (3b). 
fueron aplicados en todos los casos. Sin embargo, la VAS solamente se utilizó en 11 y la técnica SOS en 16.

En el cuadro 3 se presentan en forma resumida las descripciones de las litologías de cada uno de los sitios bajo estudio, a partir de información disponible al público por parte del Servicio Nacional de Aguas Subterráneas Riego y Avenamiento (SENARA), hasta al menos $30 \mathrm{~m}$ de profundidad medidos a partir de la superficie. Se observan casos como APMR y HCPD donde se evidencia la presencia de materiales más blandos en profundidad, situación que suele ser atípica. En algunos casos, las descripciones disponibles son poco claras y hasta contradictorias, por lo que esta información no se utilizará como criterio base para la clasificación, sino más bien como una referencia. Los sitios que no aparecen corresponden a casos donde la información de posos no está disponible.

\section{RESULTADOS}

\section{Comparación de VAA a distintas horas}

En las figuras 4, 5 Y 6, se observan sitios donde hay coincidencia entre las razones espectrales obtenidas en tres horas del día distintas: 2 am, 8 am y $3 \mathrm{pm}$. El caso de mayor similitud es AALA (Fig. 4a), donde tanto los niveles de amplitud como de máxima amplificación se asemejan mucho. Otros sitios donde se muestra esta coincidencia pero no en forma tan evidente son: SLPF (Fig. 4f) y GLIB (Fig. 6f).

También hay semejanzas entre las tres trazas pero que no muestran un pico sobresaliente en: CPAR, CCTR, CTEC, AFBR, AGRE, POSA, SJUD, CTBA, HVRG, GNYA, HHDA, LGPI y LSQR. En estos casos, la razón $\mathrm{H} / \mathrm{V}$ es cercana a la unidad en todo el rango de frecuencias, por lo que podría interpretarse como que corresponden a emplazamientos de roca. Sin embargo, esta suposición no es válida según se verá más adelante.

Emplazamientos como: SHTH, APMR, PQUE, SSNM, AORT, HPVJ y ASCS muestran gran semejanza entre las razones espectrales obtenidas con ventanas de ruido en horas del día ( 8 am y 3 pm), las cuales definen un mismo pico en forma aproximada. Sin embargo, para el caso de la medición en horas de la madrugada, la razón espectral para estos casos es casi igual a la unidad.

Esto puede deberse a que el nivel de energía de las vibraciones ambientales en esos sitios en la madrugada es tan bajo que no se excita el modo fundamental, por lo que no aparece ningún pico sobresaliente.

Se observan además sitios con razones espectrales anómalas y difíciles de interpretar como: ASRM, PJAC y HCPD. En estos, la condición geológica del emplazamiento y el nivel de energía presente en las vibraciones ambientales pudo haber provocado que su forma no tenga una posible interpretación asociada a las condiciones del suelo en el sitio.

\section{Comparación de VAA (mediciones a las 3 pm) con VAS}

Las figuras 7, 8 y 9 muestran la comparación entre las técnicas VAA y VAS, que corresponden a la medición de vibraciones ambientales con el uso de acelerógrafo y sismógrafo, respectivamente. Para el caso de VAA, se utilizaron las mediciones hechas a las $3 \mathrm{pm}$ para efectos de comparación con las pruebas VAS, debido a que es a esa hora cuando se observan picos mejor definidos en la razón espectral de VAA.

Solamente en los casos de APMR (Fig. 7c), AALA (Fig. 7a) y SLPF (Fig. 7f) se logró obtener una forma semejante entre ambas $\mathrm{H} / \mathrm{V}$, tanto en amplitud como en definición de la frecuencia predominante. En los sitios: SHTH (Fig. 7b) y SSBN (7g) se observan trazas semejantes de ambas razones espectrales pero amplitudes inferiores según la técnica VAA.

En los demás casos en los que se pudo hacer esta comparación (ver cuadro 2), las amplitudes obtenidas con VAA son mucho menores que las mostradas según VAS y en muchos casos no coinciden los picos o las mostradas por la técnica VAA tienen muy baja amplitud, cercana a la unidad, por lo que no permiten la identificación de ningún pico. Esto hace pensar que la técnica VAA con el 
Cuadro 3

Descripciones litológicas simplificadas de cada emplazamiento según el SENARA

\begin{tabular}{|c|c|c|}
\hline Estación & Profundidad (m) & Descripción litológica según pozos disponibles \\
\hline \multirow[t]{4}{*}{ AALA } & $0-18$ & Arcilla color café claro de plasticidad alta con fragmentos lávicos subredondeados y subangulares \\
\hline & $18-30$ & Toba arenosa de color café claro, formada por fragmentos líticos de composición volcánica \\
\hline & $30-36$ & Zona brechosa de composición andesítica, fracturada \\
\hline & $36-47$ & Lavas escoriáceas y brechosas \\
\hline \multirow[t]{3}{*}{ SHTH } & $0-13$ & Lavina de color café, de matriz predominantemente arcillosa sumamante densa y compacta \\
\hline & $13-20$ & Lahares compuestos por bloques decimétricos de lavas envueltos en matriz arcillosa \\
\hline & $20-36$ & Toba, matriz arcillosa meteorizada, con bloques de lava \\
\hline \multirow[t]{5}{*}{ APMR } & $0-6$ & Arcilla color café claro, muy plástica \\
\hline & $6-17$ & Toba fracturada color gris \\
\hline & $17-30$ & $\begin{array}{l}\text { Material arenoso (contiene fragmentos de lava, cuarzo, etc,) con arcilla, aparentemente se trata } \\
\text { de un depósito lacustre }\end{array}$ \\
\hline & $30-36$ & Arcilla con arena fina, color café \\
\hline & $36-40$ & Arcilla color gris \\
\hline \multirow[t]{2}{*}{ CPAR } & $0-10$ & Arcilla café claro \\
\hline & $15-45$ & Lahar, bloques de lava en matriz limo-arenosa \\
\hline \multirow[t]{2}{*}{ PQUE } & $0-15$ & $\begin{array}{l}\text { Arenisca de grano fino y color griz cafesuzco con algunos fragmentos de caliza blanca, ligera- } \\
\text { mente arcillosa y algo oxidada }\end{array}$ \\
\hline & $15-38$ & Aluvión, Su composición es mayormente sedimentaria, con lutitas y un material verde oxidado \\
\hline \multirow[t]{2}{*}{ SLPF } & $0-10$ & Arcilla café \\
\hline & $10-30$ & Lahar, Contiene arenas, arcilla, fragmentos lávicos y caolinizados \\
\hline \multirow[t]{3}{*}{ SSBN } & $0-13$ & Arcilla, color café claro, Cenizas compactadas, de grano muy fino \\
\hline & $13-31$ & $\begin{array}{l}\text { Lavina, color café claro, Con algún contenido arcilloso y una serie de fragmentos de otras } \\
\text { rocas, Entre estos fragmentos hay lavas, piroclastos, fenocristales, etc }\end{array}$ \\
\hline & $31-40$ & Toba color gris ceniciento, Con cristales de anfíboles y feldespatos en una matriz de ceniza \\
\hline \multirow[t]{2}{*}{ ASRM } & $0-10$ & Toba de granulometría fina, color café claro y muy arcillosa \\
\hline & $10-40$ & $\begin{array}{l}\text { Toba constituida por minerales y pequeños fragmentos líticos, bien cementados en una matriz } \\
\text { fina de ceniza de color café claro, algo alterada }\end{array}$ \\
\hline CCRT & $0-30$ & $\begin{array}{l}\text { Bloques centimétricos a métricos de composición heterogénea (principalmente lávico) en una } \\
\text { matriz arenosa con presencia de arcillas, Con intercalación de capas de toba lítica y capas de } \\
\text { grava, Con un espesor máximo de } 50 \mathrm{~m}\end{array}$ \\
\hline \multirow[t]{2}{*}{ CTEC } & $0-20$ & $\begin{array}{l}\text { Lahar, depósito aluvional con alto contenido de arcillas plásticas, con bloques volcánicos de } \\
\text { hasta } 1,5 \mathrm{~m}\end{array}$ \\
\hline & $20-34$ & Tobas arcillosas, color café claro con incrustaciones de lavas \\
\hline \multirow[t]{2}{*}{ AFBR } & $0-34$ & Arcillas café con bloques de gran tamaño \\
\hline & $34-70$ & Tobas limosas de lava e ignimbrita \\
\hline \multirow[t]{2}{*}{ AGRE } & $0-12$ & Toba constituida por ceniza fina de color café oscuro, arcillosa \\
\hline & $12-60$ & Ceniza media a gruesa de color negro y aspecto arenoso \\
\hline \multirow[t]{2}{*}{ PJAC } & $0-4$ & Arcillas \\
\hline & $4-9$ & $\begin{array}{l}\text { Arenas finas intercaladas con arcillas en capas típicas de rellenos aluviales, de granulometría } \\
\text { fina a media y de mala selección }\end{array}$ \\
\hline
\end{tabular}


Cuadro 3 (continuación)

Descripciones litológicas simplificadas de cada emplazamiento según el SENARA

\begin{tabular}{|c|c|c|}
\hline Estación & Profundidad (m) & Descripción litológica según pozos disponibles \\
\hline \multirow[t]{2}{*}{ PJAC } & $9-15$ & Gravas aluviales en matriz arenosa \\
\hline & 15 & Basamento rocoso basáltico \\
\hline \multirow[t]{4}{*}{ POSA } & $0-2$ & Arcilla \\
\hline & $2-17$ & Limolita meteorizada, color marrón con láminas de estratificación \\
\hline & $17-18$ & Caliza, color crema, con impurezas, maciza sana \\
\hline & $18-27$ & Arenisca, color gris oscuro, grano fino \\
\hline \multirow[t]{6}{*}{ SJUD } & $0-3$ & Materiales arcillosos \\
\hline & $3-9$ & $\begin{array}{l}\text { Toba meteoriza, matriz color café oscuro, con fragmentos claros o tobáceos, otros café claro } \\
\text { (arcillificados) }\end{array}$ \\
\hline & $9-32$ & Toba pumítica, color uniforme gris claro, dureza mediana \\
\hline & $32-35$ & $\begin{array}{c}\text { Toba meteorizada, pequeños fragmentos de vidrio volcánico (negros), en matriz café clara, } \\
\text { deleznable }\end{array}$ \\
\hline & $35-37$ & Toba arenosa, fragmentos frescos oscuros en un $85 \%$ y el $15 \%$ claros, \\
\hline & $37-43$ & $\begin{array}{l}\text { Toba pumítica, compuesta de pómez, Es abrasiva al tacto, color gris claro, Similar de } 9 \text { a 32m, } \\
\qquad \text { El contenido de arcilla es bajo }\end{array}$ \\
\hline \multirow[t]{4}{*}{ CTBA } & $0-3$ & Suelos limo-arcillosos \\
\hline & $3-13$ & $\begin{array}{l}\text { Aluvión con cantos subangulares, de lutitas y rocas sedimentarias, envueltos en una matriz } \\
\text { areno-arcillosa, Las arenas son de grano grueso }\end{array}$ \\
\hline & $13-18$ & Aluvión, Gravas y cantos rodados, envueltos en una matriz areno-arcillosa de grano medio \\
\hline & $18-31$ & Aluvión, Estrato con arenas medias, arenas arcillosas, con cantos rodados subangulares \\
\hline \multirow[t]{7}{*}{ HPVJ } & $0-12$ & Arcilla; color café, densa, plástica con un contenido de materia orgánica alto \\
\hline & $12-19$ & $\begin{array}{c}\text { Arcilla con arena en proporción 4:1, Los fragmentos de arena, medianamente redondeadas son } \\
\text { tobáceas }\end{array}$ \\
\hline & $19-20$ & $\begin{array}{l}\text { Arcilla con grava, en proporción 3:1, Los fragmentos de grava semiredondeados son de origen } \\
\text { tobáceos }\end{array}$ \\
\hline & $20-21$ & Arcilla con arena, en proporción 5:1 \\
\hline & $21-23$ & Arcilla con arena, en proporción 2:1, los fragmentos tobáceos \\
\hline & $23-29$ & $\begin{array}{l}\text { Grava con poca arcilla, Setrata de una grava fina, tobácea de fragmentos poco meteorizados y } \\
\text { abundante sílice amorfo (vidrio) }\end{array}$ \\
\hline & 29-36 & $\begin{array}{l}\text { Toba color café, algo meteorizada, matriz arcillosa, Abundantes fragmentos líticos en matriz cin- } \\
\text { erítico-vítrea, Hay perlita y otros silicatos amorfos secundarios que le restan porosidad al conjunto }\end{array}$ \\
\hline \multirow[t]{3}{*}{ HCPD } & $0-4$ & Arcilla café-grisaceo, liviana, húmeda se torna adhesiva \\
\hline & $4-24$ & $\begin{array}{l}\text { Lahar arcilloso de color café amarillento, incluye secciones con clastos sub-angulares y sub- } \\
\text { redondeados de lava, masa feldespática, granos de cuarzo amorfo, piezas caolinizadas }\end{array}$ \\
\hline & $24-36$ & Arcilla de color amarillento, dura, incluye algunos fragmentos de lava \\
\hline \multirow[t]{2}{*}{ AORT } & $0-28$ & $\begin{array}{l}\text { Material coluvio-aluvial: formado por fragmentos de diferente forma, tamaño y composición, } \\
\text { englobados caóticamente en una matriz arcillosa de color café claro a rojizo }\end{array}$ \\
\hline & $28-50$ & $\begin{array}{l}\text { Toba gris conformada por fragmentos líticos y minerales, bien cementados por una matriz fina } \\
\text { de color gris, Ligeramente alterada }\end{array}$ \\
\hline
\end{tabular}


Cuadro 3 (continuación)

Descripciones litológicas simplificadas de cada emplazamiento según el SENARA

\begin{tabular}{|c|c|c|}
\hline Estación & Profundidad (m) & Descripción litológica según pozos disponibles \\
\hline GLIB & $0-36,6$ & $\begin{array}{l}\text { Toba blanca riolítica bien cementada, cuarzosa, De la Formación Liberia incluye pocos frag- } \\
\text { mentos de toba café, láminas de mica meteorizada, Algunos cristales fémico negros, muchos } \\
\text { cristales de cuarzo, El material piroclástico va decreciendo con la profundidad }\end{array}$ \\
\hline \multirow[t]{3}{*}{ GNYA } & $0-10$ & Arcillas \\
\hline & $10-20$ & Material sedimentario: Lutitas y areniscas de grano fino a medio \\
\hline & $20-40$ & Basaltos medios a sanos del Complejo de Nicoya \\
\hline \multirow[t]{2}{*}{ HHDA } & $0-7,7$ & Suelos arcillosos y limoarenosos, proveniente de la meteorización de materiales piroclásticos \\
\hline & $7,7-30,2$ & $\begin{array}{c}\text { Formación Barva: constituida por lavas fracturadas, andesíticas, brechas lávicas, lavas densas } \\
\text { y duras en la sección superior de esta secuencia lávica, y más fracturadas, pero la sección es } \\
\text { dominada por tobas }\end{array}$ \\
\hline \multirow[t]{4}{*}{ LGPI } & $0-8$ & Aluvión grande con arcilla \\
\hline & $8-18$ & Aluvión mediano sin contenido de arcilla \\
\hline & $18-28$ & Aluvión limpio sin arcilla \\
\hline & $28-34$ & Aluvión con poca arcilla \\
\hline \multirow[t]{2}{*}{ LSQR } & $0-4$ & $\begin{array}{l}\text { Material heterogéneo muy meteorizado, La parte superior está formada de un suelo, formado a } \\
\text { partir de un aluvión }\end{array}$ \\
\hline & $4-28$ & $\begin{array}{c}\text { Este aluvión está constituido por fragmentos lávicos principalmente, y en menor proporción, } \\
\text { se encuentran pedernales de coloración verdosa y rojiza y fragmentos de toba, Estos materiales } \\
\text { están redondeados, Cabe destacar que dichos materiales se encuentran contaminados de finos } \\
\text { (arcillas y limo) }\end{array}$ \\
\hline \multirow[t]{3}{*}{ AFRA } & $0-4$ & Arcilla café claro \\
\hline & $4-23,5$ & Lava (densa), rojiza con negro y fracturada \\
\hline & $23,5-47,6$ & 23,5-47,6: Lava (densa), color verde claro y pálido \\
\hline
\end{tabular}

uso de acelerógrafos, no es muy confiable para identificar las frecuencias naturales de vibración de un depósito de suelo, tal y como lo han demostrado otros autores como Mucciarelli (1998).

\section{Comparación de VAA con ST}

Las mismas figuras 4, 5 y 6 muestran las comparaciones entre las técnicas VAA (vibraciones ambientales con acelerógrafo) y ST (registros de sismos totales obtenidos con acelerógafos).

Se evidencian algunos casos en los que las razones espectrales son semejantes, solo que las obtenidas con la técnica VAA tienen amplitudes menores, aunque sí logran definir la misma frecuencia correspondiente a la de mayor amplificación. Los sitios que cumplen con esta descripción son: AALJ, SHTH, SLPF, y GLIB. Por otro lado SSBN, HPVJ, ASCS, GNYA, HHDA, PQUE y HVRG muestran posibles efectos de no linealidad del suelo ya que las frecuencias identificadas con ST son menores que las observadas con VAA. Esto se sustenta en el hecho de que el movimiento producido por los sismos siempre es más intenso que el debido a vibraciones ambientales, de ahí que las frecuencias disminuyan al ser el medio sometido a una sacudida más fuerte como consecuencia de una "flexibilización " del suelo debido a su comportamiento no lineal.

En varios casos se observa que el nivel de amplificación obtenido con VAA es muy plano y cercano a la unidad en comparación con ST, 
AALA

—VAA 02:00 a.m. ‥VAA 03:00 p.m. — VAA 08:00 a.m.

a)
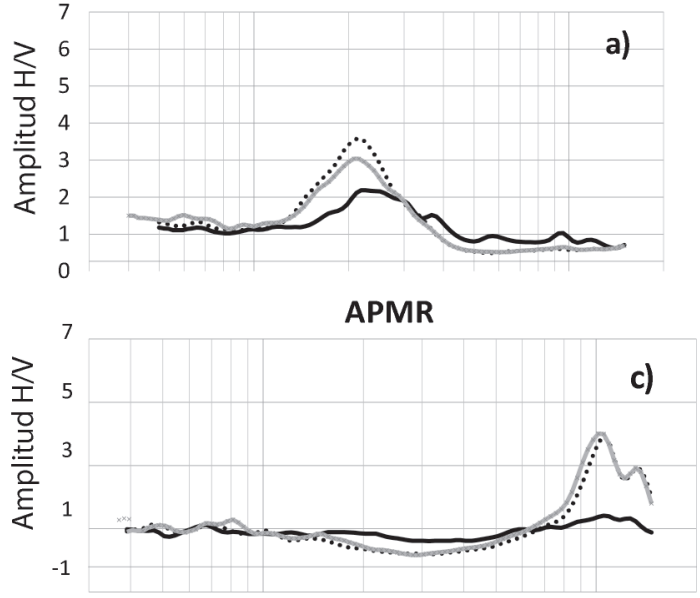

PQUE

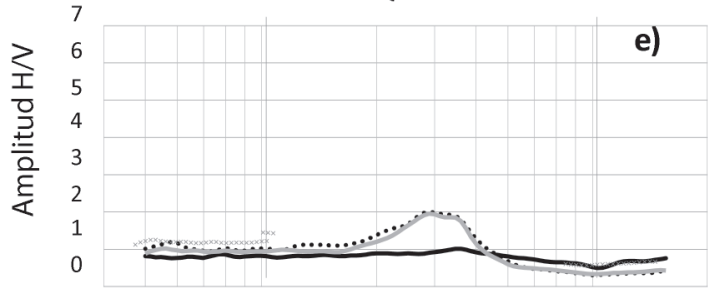

SSBN
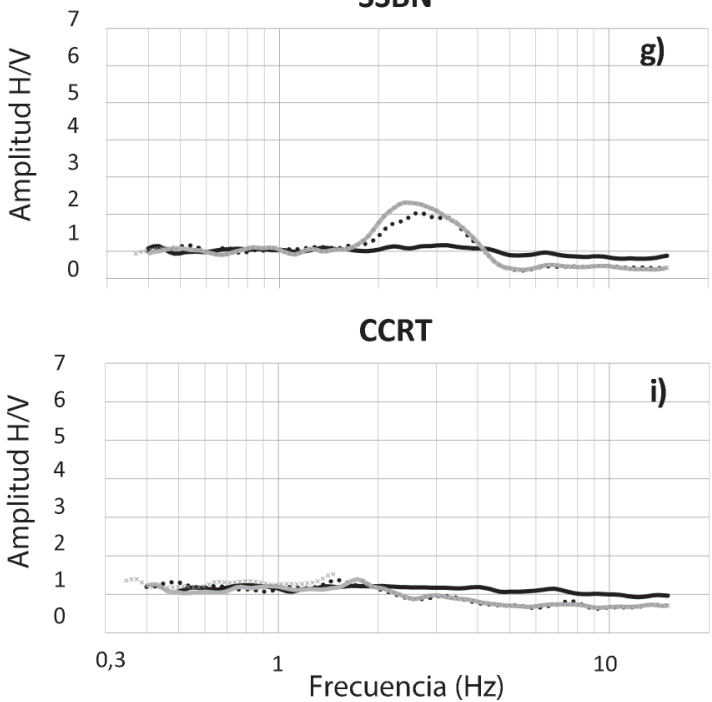

SHTH

— VAA 01:30 a.m. $\cdots$ VAA 03:00 p.m. - VAA 08:00 a.m.

b)

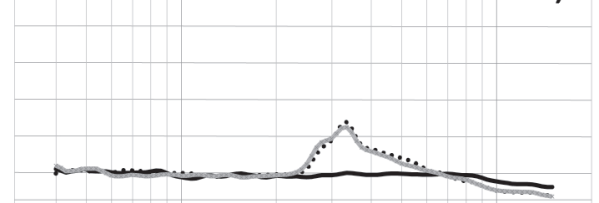

CPAR

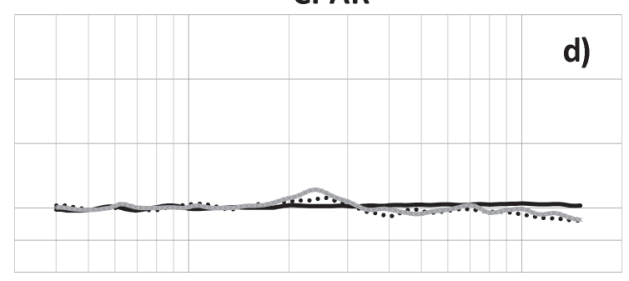

SLPF

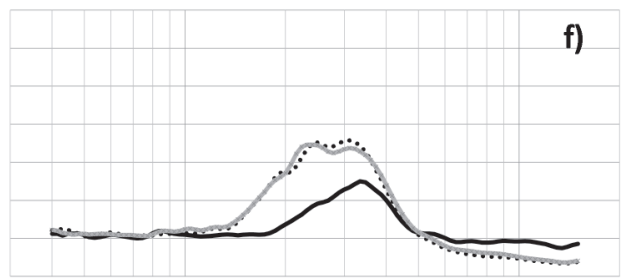

ASRM

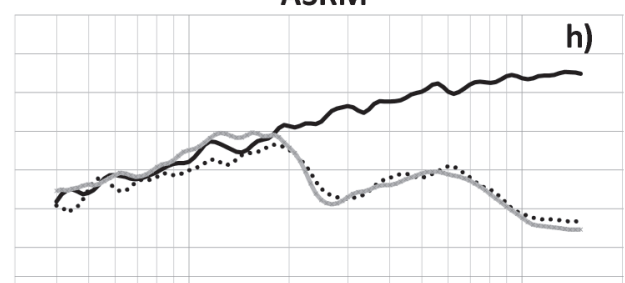

CTEC

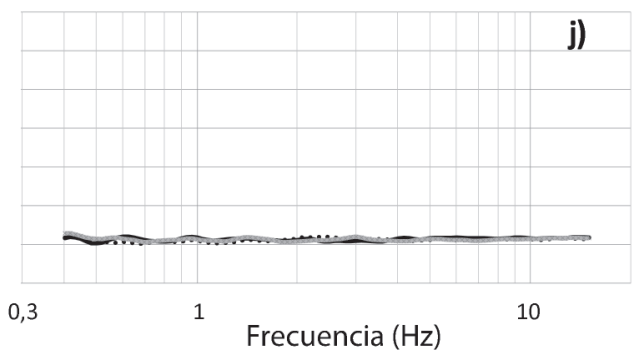

Fig. 4: Aplicación de la técnica VAA a tres horas distintas: 2 am, 8 am y 3 pm en los siguientes sitios: AALA, SHTH, APMR, CPAR, PQUE, SLPF, SSBN, ASRM, CCRT y CTEC. 
AFBR

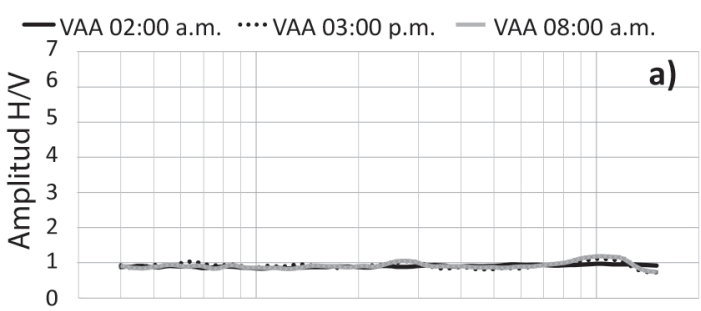

PJAC

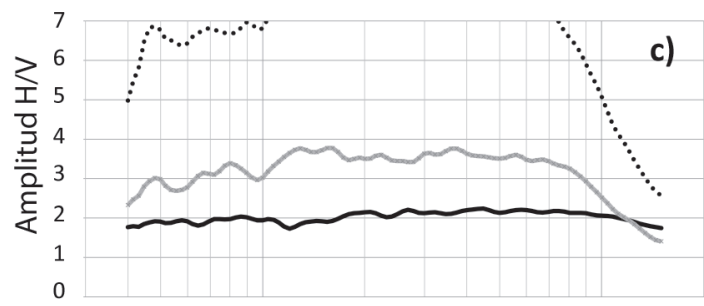

SJUD

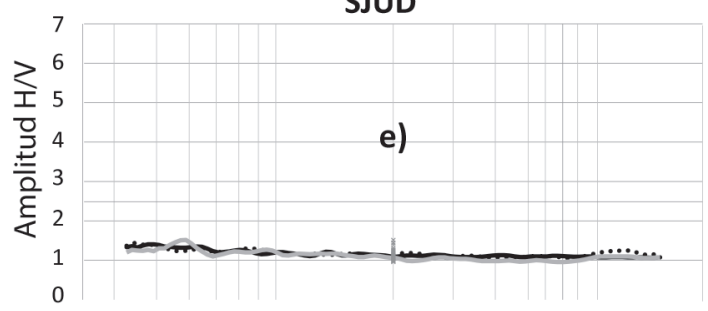

HPVJ

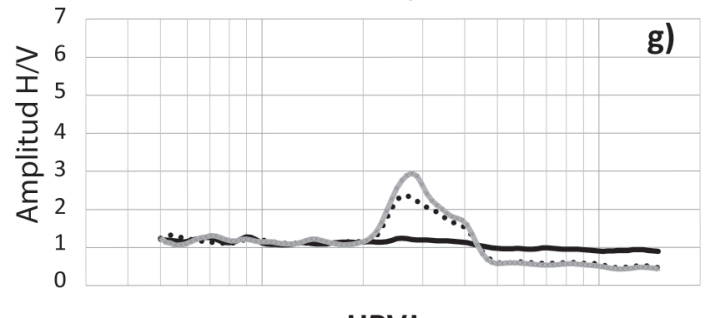

HPVJ

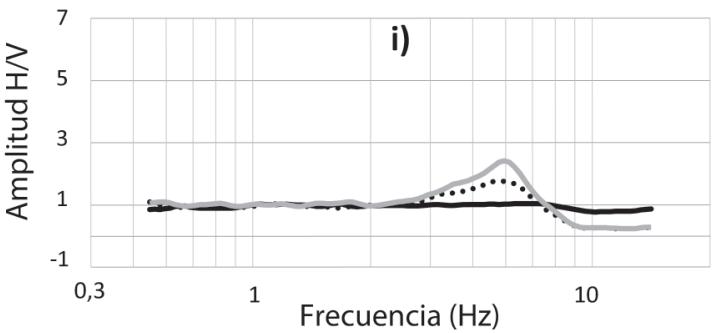

AGRE

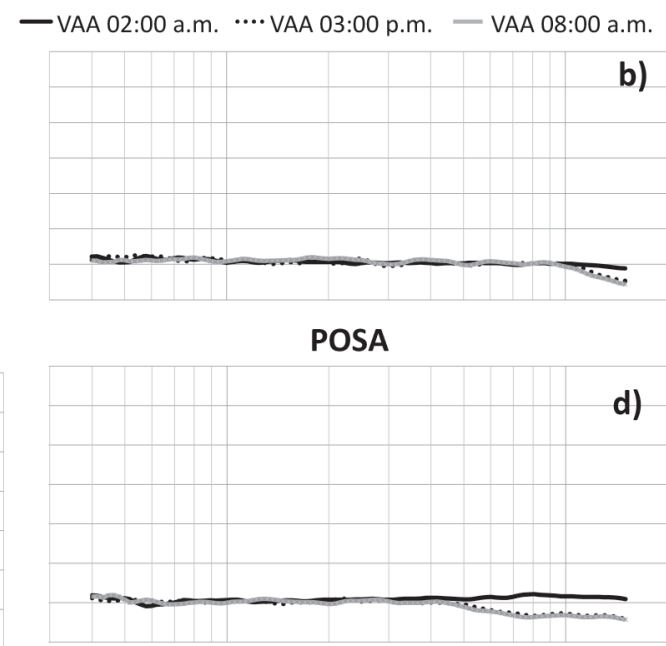

CTBA

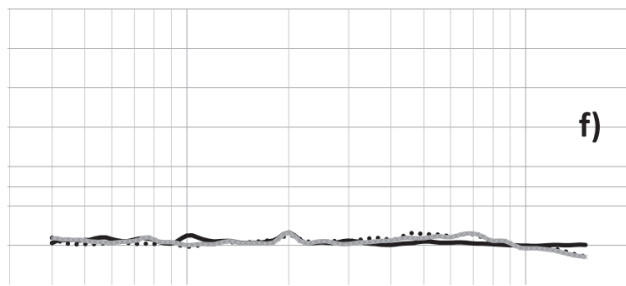

HVRG

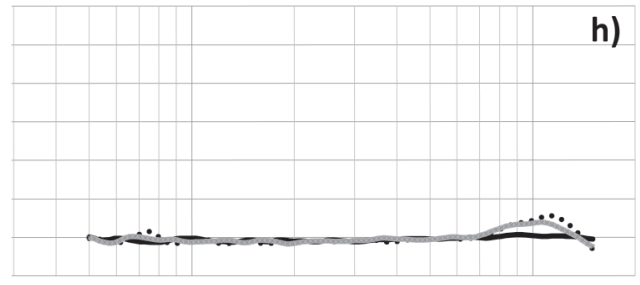

HVRG

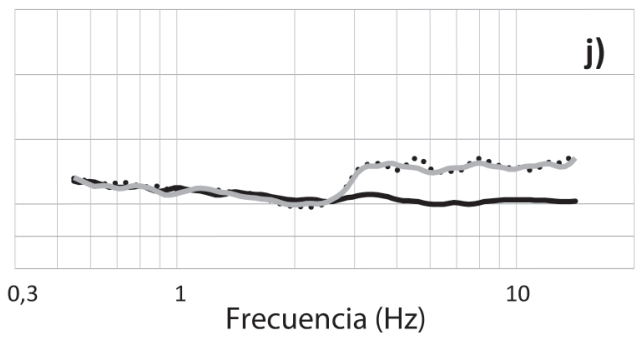

Fig. 5: Aplicación de la técnica VAA a tres horas distintas: 2 am, 8 am y 3 pm en los siguientes sitios: AFBR, AGRE, PJAC, POSA, SJUD, CTBA, HPVJ, HVRG, ASCS y HCPD. 
AORT
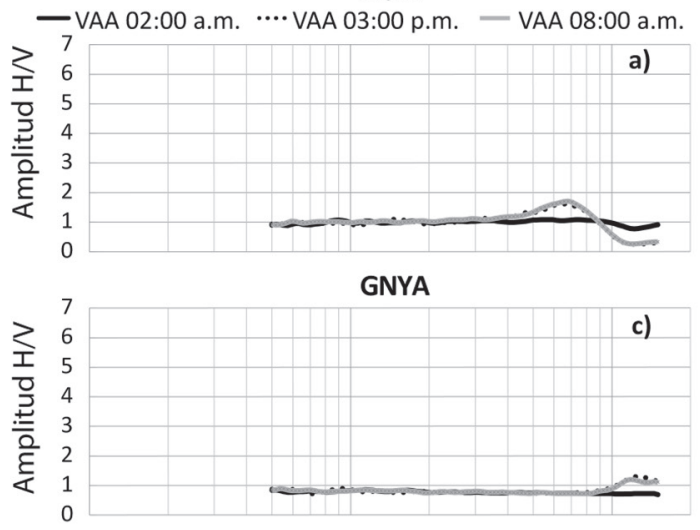

LGPI

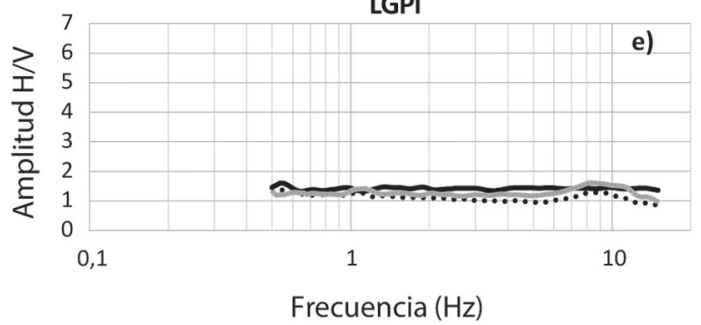

GLIB

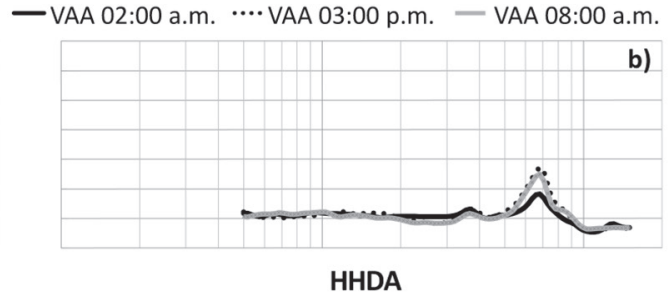

b)

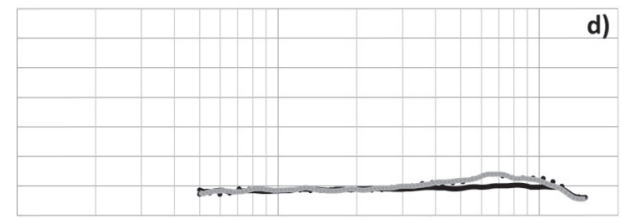

LSQR

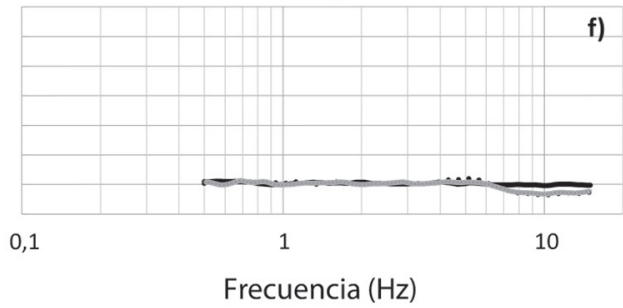

Fig. 6: Aplicación de la técnica VAA a tres horas distintas: 2 am, 8 am y 3 pm en los siguientes sitios: AORT, GLIB, GNYA, HHDA, LGPI y LSQR.

que si define mucho mejor los picos. Esto es de esperarse ya que provienen de tipos de movimiento (niveles de energía) muy distintos. Los sitios que muestran estas características son: CPAR, ASRM, CCRT, CTEC, AFBR, AGRE, POSA, SJUD, CRBA, LGPI y LSQR.

También se observan sitios donde no existe coincidencia entre los picos visibles. Estos son: APMR, PJAC, HCPD Y AORT, que además son emplazamientos con geologías más complejas que las demás, como es el caso de APMR, en el que incluso se evidencia una inversión de velocidades de la onda cortante (Schmidt, 2014, Rojas, 2013).

Debido a tal complejidad y a la corta trayectoria que siguen las ondas superficiales originadas por las vibraciones ambientales hasta alcanzar la superficie, que además contienen poca energía respecto a las ondas producidas por sismos, es de esperarse que la técnica ST aporte información más detallada de las distintas capas que forman un perfil de suelos, por lo que se logra definir de mejor manera las irregularidades y posibles anomalías presentes en dicho perfil. Debe hacerse notar que las ondas producidas por sismos viajan mayores distancias y provienen de profundidades mucho mayores que las de origen ambiental, por lo que contienen mayor cantidad y calidad de información del subsuelo.

\section{Comparación de VAS con ST}

Esta comparación solamente se pudo llevar a cabo en 12 sitios, que fue en los que se logró trasladar un sismógrafo portátil y colocarlo al lado del acelerógrafo que se encuentra fijado permanentemente. En las figuras 7 y 8 se observa que en la mayoría de los sitios (AALA, CPAR, SLPF, SSBN, CTEC, AFBR y AGRE) muestran formas 
AALA
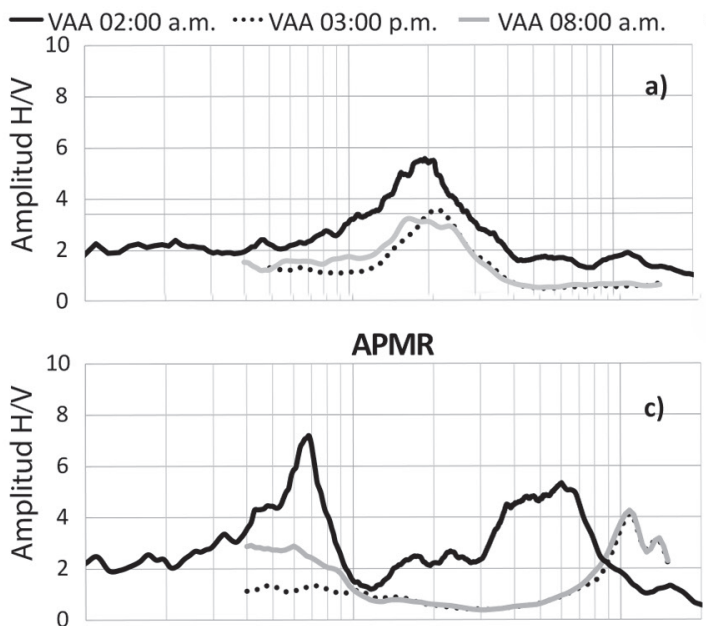

PQUE

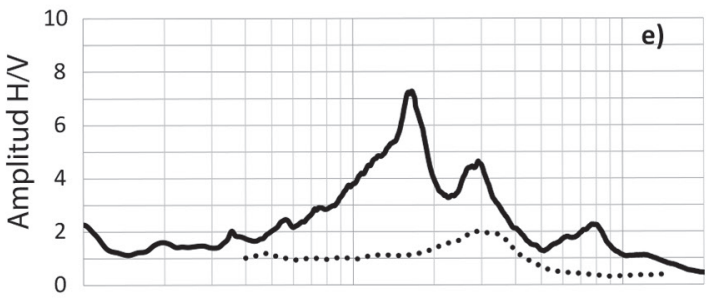

CCRT

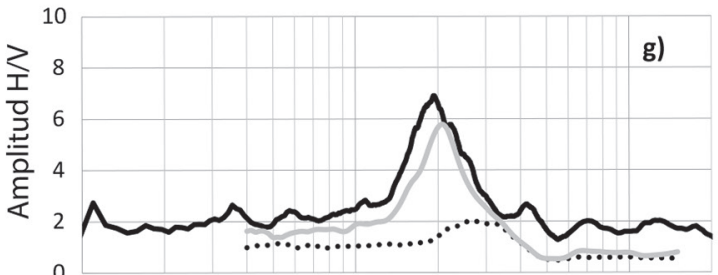

SSBN

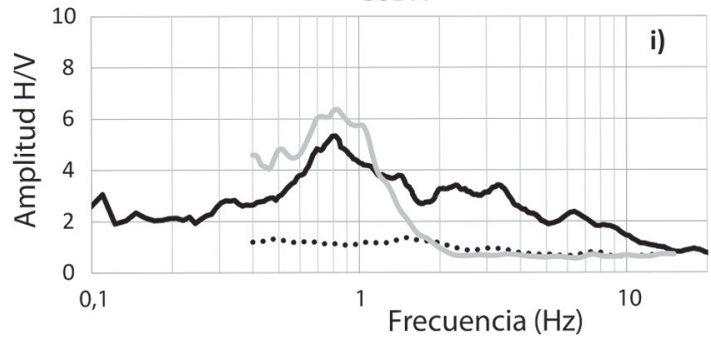

SHTH

—VAA 02:00 a.m. …VAA 03:00 p.m. — VAA 08:00 a.m.

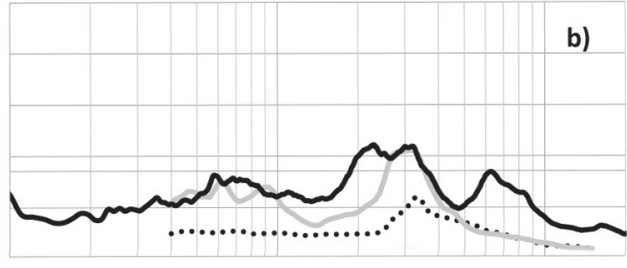

CPAR

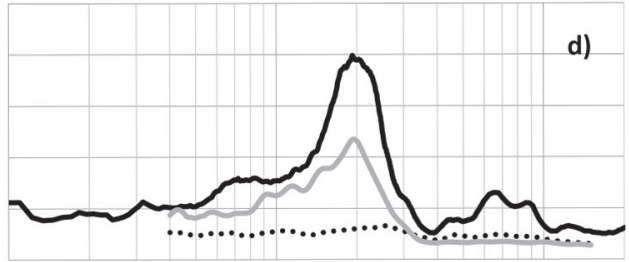

SLPF

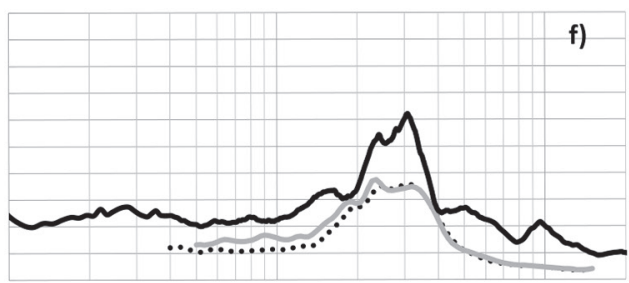

CTEC

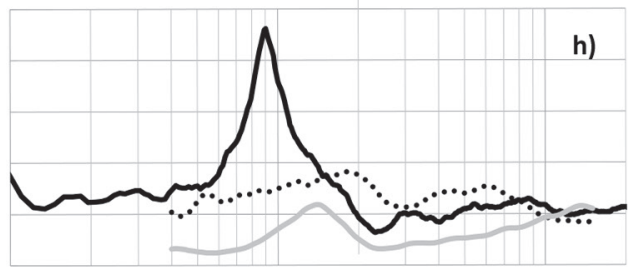

ASRM

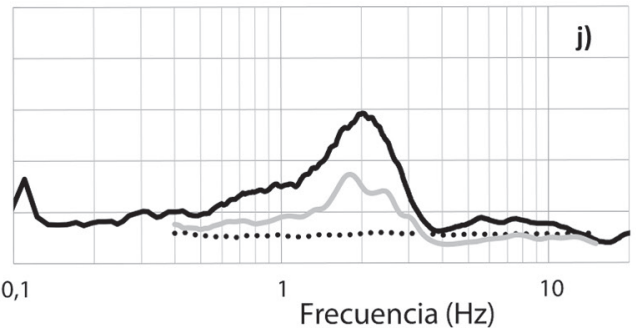

Fig. 7: Comparación entre las técnicas VAA a las 3 pm (línea punteada), VAS (línea gris) y ST (línea negra) en los sitios: AALA, SHTH, APMR, CPAR, PQUE, SLPF, SSBN, ASRM, CCRT y CTEC. 
AFBR

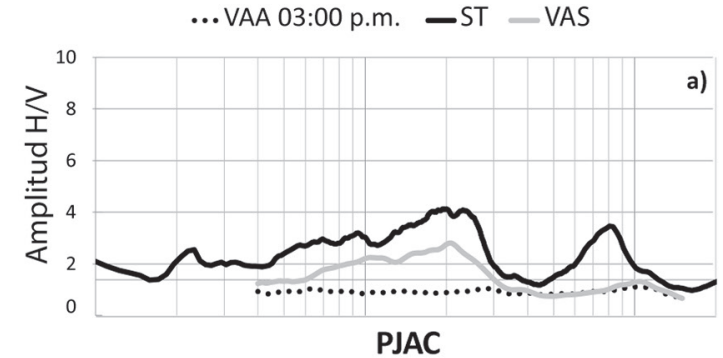

...VAAA 03:00 p.m. - - ST

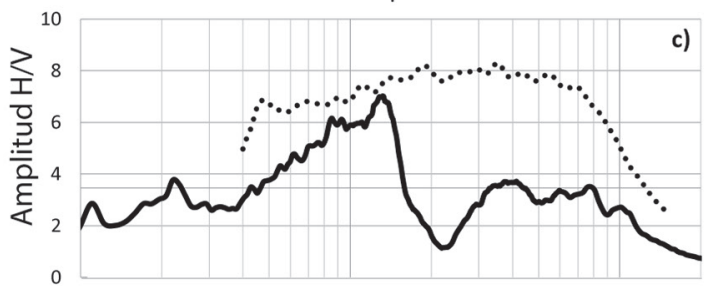

SJUD

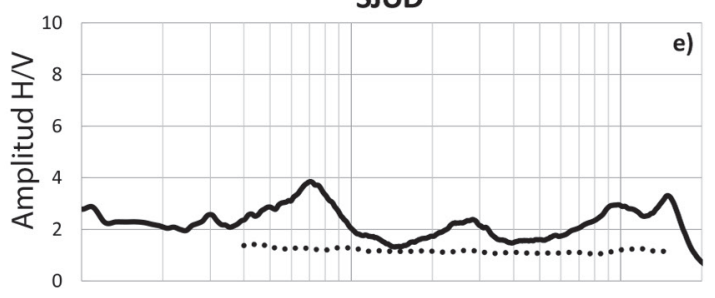

HPVJ
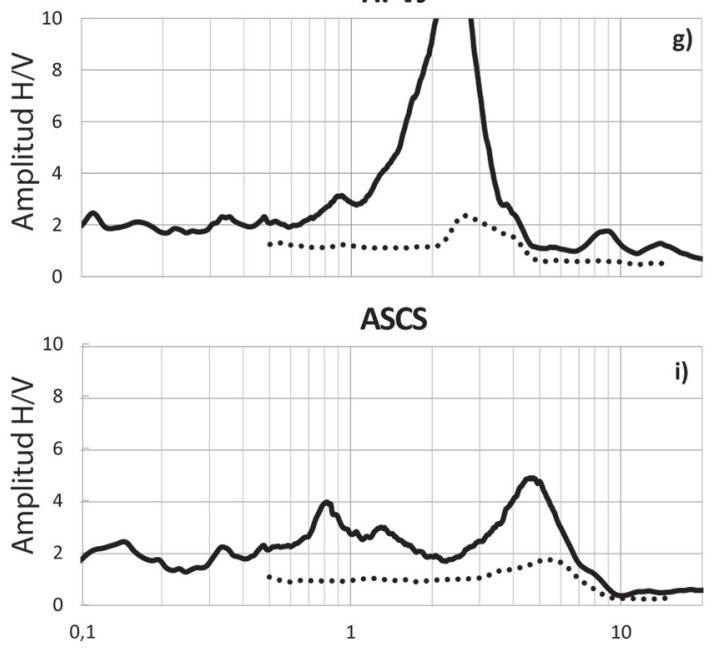

Frecuencia $(\mathrm{Hz})$
AGRE

...VAA 03:00 p.m. —ST — VAS

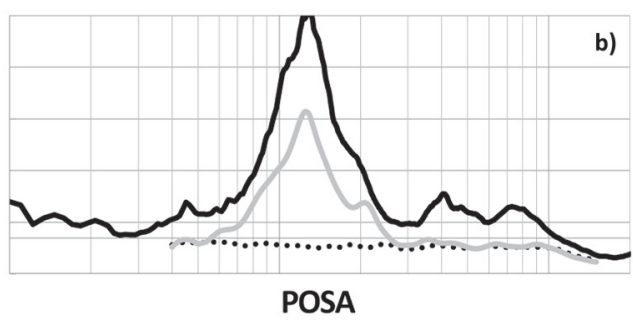

...VAA 03:00 p.m. - - ST

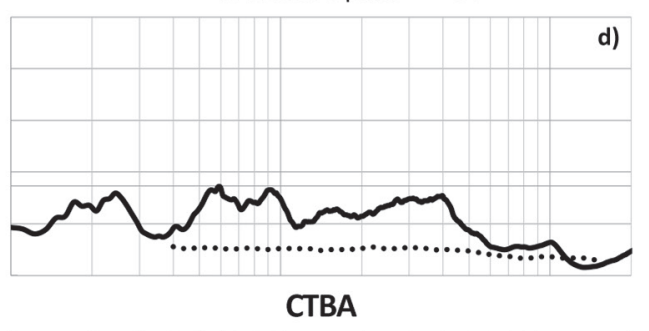

f)

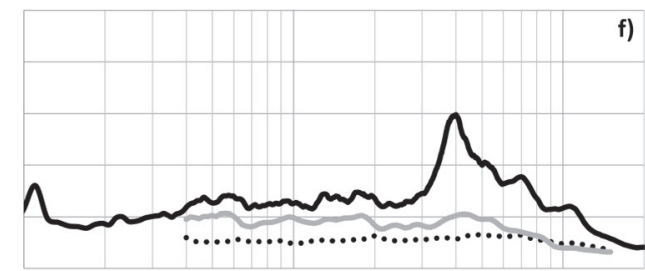

HVRG

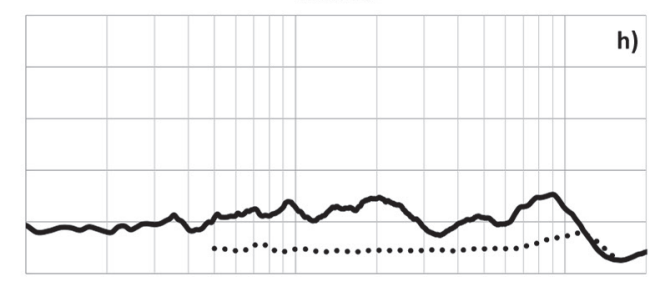

HCPD

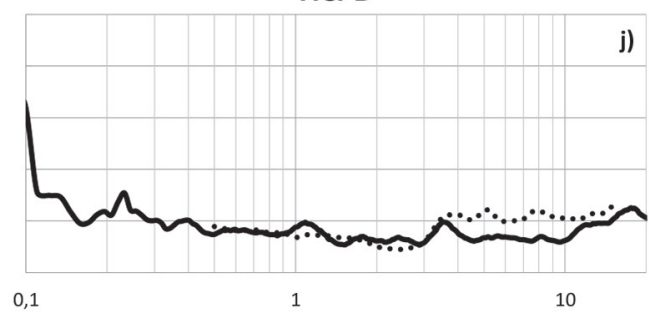

Frecuencia $(\mathrm{Hz})$

Fig. 8: Comparación entre las técnicas VAA a las 3 pm (línea punteada), VAS (línea gris) y ST (línea negra) en los sitios: AFBR, AGRE, PJAC, POSA, SJUD, CTBA, HPVJ, HVRG, ASCS y HCPD. 
semejantes entre ambas razones espectrales, pero con menor amplitud las obtenidas con la técnica VAS, lo que era de esperarse ya que son ondas que tienen un nivel de energía mucho menor que las generadas por sismos, debido a las razones expuestas en párrafos anteriores.

Otros sitios muestran las siguientes singularidades:

SHTH (Fig. 7b): se manifiesta un aparente segundo modo en ambas razones espectrales y los niveles de amplificación son semejantes entre ellas.

APMR (Fig. 8a): no existe ninguna coincidencia entre ambas razones espectrales, debido probablemente a las irregularidades del subsuelo en ese sitio (ver cuadro 3).

ASRM (Fig. 7h): la técnica ST muestra mayores amplificaciones respecto a VAS, como sucede en la mayoría de los sitios, pero en este caso se aprecian posibles efectos de no linealidad del suelo ya que ST muestra menor frecuencia fundamental que VAS, que corresponde a mediciones de menor energía porque provienen de vibraciones ambientales, En este sitio, la presencia significativa de suelo blando (entre S3 y S4 según Schmidt, 2014) justifica su comportamiento no lineal del medio cuando es sometido a vibraciones intensas, como son los sismos.

CCRT (Fig. 7i): a pesar de que ambas técnicas definen la misma frecuencia fundamental, contrario a los casos anteriores VAS supera en amplitud a ST en un rango de frecuencias de 0,4 a $1,5 \mathrm{~Hz}$.

CTBA (Fig. 8f): VAS muestra niveles de amplificación muy bajos y semejantes entre ellos, cercanos a 2, mientras que ST llega a una amplitud de 6 en la frecuencia fundamental. Esto puede deberse a que un contraste significativo entre estratos, que es el responsable de generar amplificaciones significativas de la frecuencia fundamental (Kramer, 1996) existe a profundidades mayores que las que las ondas superficiales logran llegar, por lo que sí se observa claramente en ST (sismos) y no en VAS (vibraciones ambientales).

\section{Comparación entre ST y SOS}

Se decidió hacer esta comparación ya que, a pesar de que se obtuvieron a partir de los mismos registros, según Rielp et al (1998) resulta ser más efectiva la definición del espectro de amplificación para una ventana de 5 segundos a partir del arribo de las ondas $\mathrm{S}$, que con el uso del registro completo. El uso de esta ventana permite obtener tal función en la zona más intensa del registro y con una cantidad de cálculos mucho menor que si se usara el registro total.

En este estudio se encontró que de los 15 sitios donde se hizo la comparación, (ver figuras 10 y 11) en 10 de ellas hay una coincidencia casi perfecta en la forma de ambas funciones.

Los siguientes sitios muestran algunas singularidades: AFBR, AGRE, PJAC, POSA y PJAC. Todos ellos difieren levemente su amplitud en frecuencias inferiores a $0,9 \mathrm{~Hz}$ pero definen en forma semejante la frecuencia fundamental de cada sitio, que normalmente se ubica en frecuencias superiores a ese valor, por lo que no afecta su identificación si se utiliza una técnica u otra. Solamente el caso de SJUD (Fig. 11e) muestra, además de este rango de frecuencias, diferencias significativas en amplitudes en un rango de 2 a $3 \mathrm{~Hz}$, probablemente asociadas a la inestabilidad de los materiales característicos de ese emplazamiento (ver cuadro 3 ).

Al contrastar los resultados de la aplicación de las técnicas antes mencionadas con las descripciones litológicas, se pueden definir tres sitios en los que la amplificación es casi nula en todo el rango de frecuencias debido a los tipos de suelo predominantes.

Según el cuadro 3, en GLIB existe una toba blanda bien cementada en toda la profundidad considerada (36,6 m), algo semejante a lo que sucede en SJUD a partir de los $3 \mathrm{~m}$.

En HVRG no aparecen picos de amplitud considerable en la razón espectral, a pesar de que predomina un suelo blando (arcillas de distintas características hasta los $29 \mathrm{~m}$, ver cuadro 3). Esto es 
concordante con el hecho de que, al no haber picos significativos en la razón $\mathrm{H} / \mathrm{V}$, no se evidencia un contraste significativo de impedancias en los materiales, lo cual queda claramente justificado en las descripciones litológicas del cuadro mencionado.

\section{DISCUSIÓN}

La técnica VAA (vibraciones ambientales medidas con acelerógrafos) fue aplicada en 26 sitios, de los cuales solamente en un $11,5 \%$ se obtuvo resultados semejantes en las tres distintas horas en las que se hicieron mediciones. En un $50 \%$ de los emplazamientos la amplificación fue igual a la unidad (no se evidenció amplificación). Por otro lado, se observaron amplificaciones más altas en horas del día ( 8 am o 3 pm) en un $27 \%$ de los casos y razones espectrales anómalas (formas extrañas) en un $11,5 \%$ de los sitios.

De lo anterior, se pude afirmar que lo más conveniente es aplicar esta técnica en horas del día; sin embargo, no es muy confiable el uso de acelerógrafos debido a que en general, en nivel de amplificación que se obtiene es muy bajo, debido probablemente a limitaciones de resolución de este tipo de equipos, que en muchos casos no son diseñados para registrar vibraciones débiles como las ambientales.

Al comparar las técnicas de VAA aplicada a registros de las $3 \mathrm{pm}$ con la de VAS (vibraciones ambientales con sismógrafo) en 12 de los sitios bajo estudio se obtuvo lo siguiente: un $25 \%$ muestran razones espectrales con formas y amplitudes semejantes, un 17\% exhiben formas semejantes y amplitudes distintas y un 58\% presentan formas y amplitudes distintas.

Con base en lo anterior, el uso de acelerógrafos para la determinación de la frecuencia fundamental del sitio no puede ser validado, ya que se toma como adecuado el uso de sismógrafos para una adecuada identificación de este parámetro (Mucciarelli, 1998).

Se compararon además las técnicas VAS con ST en 12 sitios y en un $75 \%$ se observan coincidencias, aunque la VAS muestra inferiores niveles de amplitud. Por lo tanto, para la identificación aproximada de la frecuencia fundamental, si se puede utilizar la VAS como aproximación de la ST, tal y como lo propone Nakamura (1989), lo que implica una forma sencilla y práctica de lograrlo, ya que puede obtenerse en cualquier momento y con mediciones de corta duración. Sin embargo, debe recordarse que no siempre se logran resultados adecuados, principalmente cuando no hay un contraste significativo entre impedancias o este es muy profundo, tal y como se mencionó antes, por lo que la VAS debe aplicarse con cautela y preferiblemente combinarla con otras técnicas.

La comparar las técnicas de ST (sismo total) con SOS (sismo onda S) en 12 sitios, se logra observar que el $94 \%$ de los casos muestran una forma muy semejante entre las razones espectrales, así como la misma definición del pico máximo, por lo que la aplicación de una u otra técnica es indiferente, Sin embargo, la SOS implica un poco más de trabajo para definir visualmente o por medio de algoritmos el arribo de la onda S, por lo que su aplicación se vuelve más lenta, aún cuando se requiere una menor cantidad de cálculos para su aplicación ya que las ventanas utilizadas son de menor duración.

\section{CONCLUSIONES}

Debido a que $\mathrm{f}_{0}$ ( o su inverso $\mathrm{T}_{0}$ ) son parámetros muy valiosos para caracterizar un emplazamiento, se investigó sobre varias técnicas que permiten obtenerlos en forma rápida y a un costo moderado.

Todas se basan en la obtención de la razón espectral H/V de los espectros de Fourier obtenidos a partir de los registros de sismos o de vibraciones ambientales, con el uso de acelerógrafos o de sismógrafos como instrumentos de medición.

Se utilizaron cuatro técnicas: VAA y VAS que corresponden al registro en el tiempo de vibraciones ambientales con el uso de acelerógrafos o sismógrafos, respectivamente. Por otro lado, se aplicaron ST y SOS, que provienen del registro completo del sismos (ST) y del uso de la porción del registro cinco segundos después del arribo de las ondas S (SOS). 
AORT
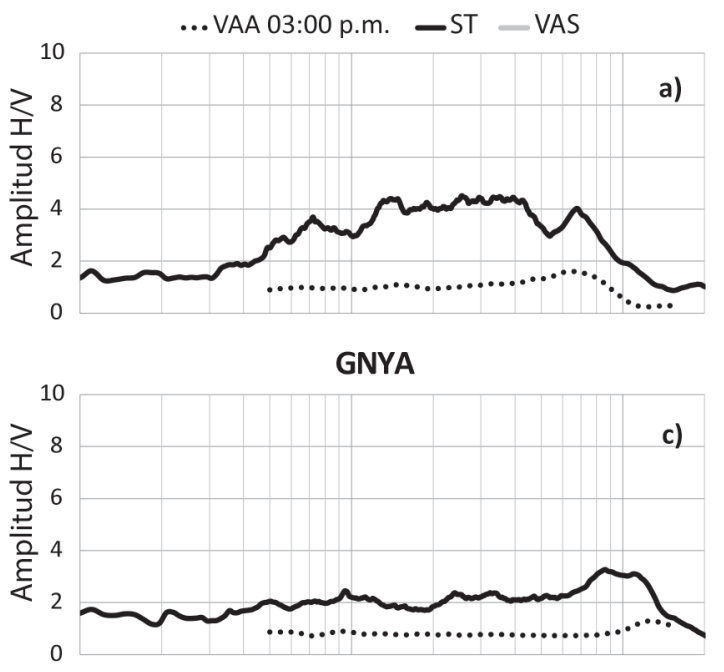

LGPI

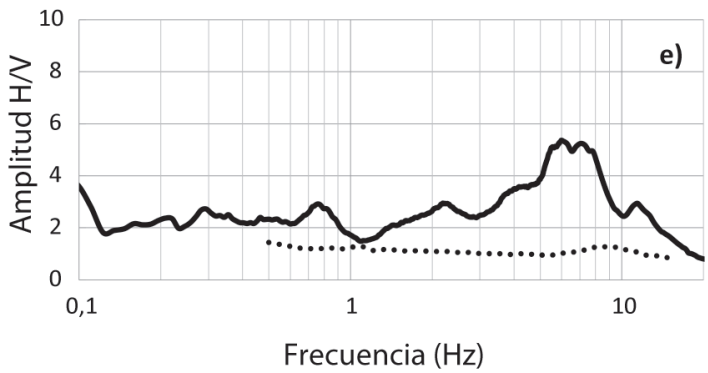

GLIB

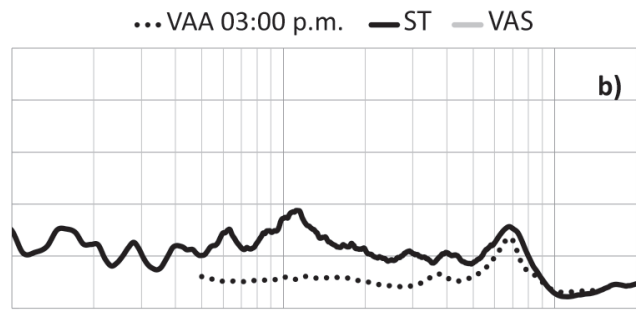

HHDA
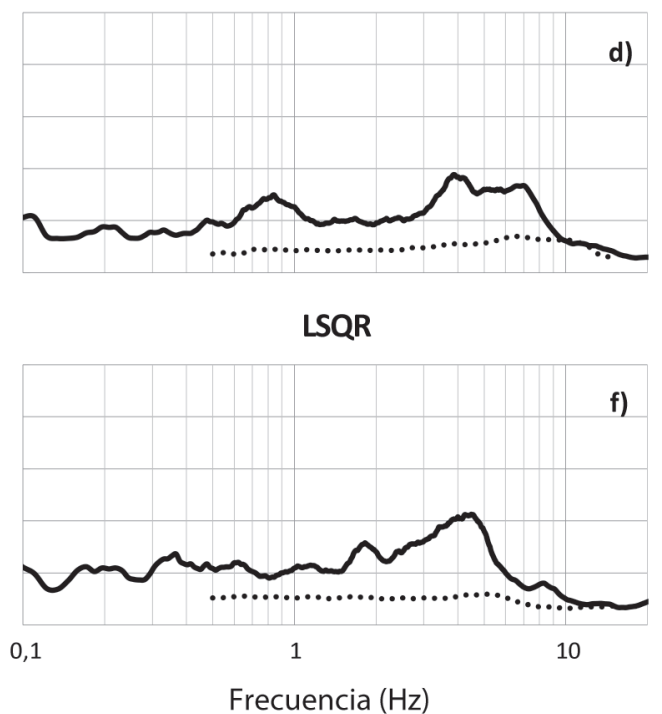

Fig. 9: Comparación entre las técnicas VAA a las 3 pm (línea punteada) y ST (línea negra) en los sitios: AORT, GLIB, GNYA, HHDA, LGPI y LSQR.

La VAA se aplicó para tres horas del día distintas: 2 am, 8 am y 3 pm. A pesar de evidenciarse niveles de amplificación y formas distintas en varios de los 26 casos estudiados, fueron las mediciones hechas a las $3 \mathrm{pm}$ las que mostraron una mejor definición de las razones espectrales. Lo anterior debido probablemente a que el nivel de energía de las vibraciones ambientales a las otras horas del día es muy bajo, por lo que no se logran identificar picos sobresalientes en el gráfico.

En sitios como AALA se obtuvo coincidencia entre las razones espectrales obtenidas por las distintas técnicas y en otros como PJAC y HCPD las formas de estos gráficos son difíciles de interpretar, debido probablemente a la presencia de una geología compleja.
Al comparar VAA con VAS, 5 de los 12 casos considerados son muy similares en todo el rango de frecuencias. Estos sitios son: APMR, AALA, SLPF, SHTH y SSBN.

Las técnicas VAA y ST muestran semejanzas en las formas de las razones espectrales, aunque las obtenidas con VAA muestras niveles de amplificación inferiores. En algunos casos como SSBN, HHDA y ASCS se observan posibles efectos de no linealidad del suelo, ya que la frecuencia fundamental del depósito $\mathrm{f}_{0}$ que muestra ST es inferior a la identificada con VAA que corresponde a vibraciones ambientales (movimiento mucho menos intenso que los sismos), por lo que la frecuencia cambia. 
AALA

- SOS - ST

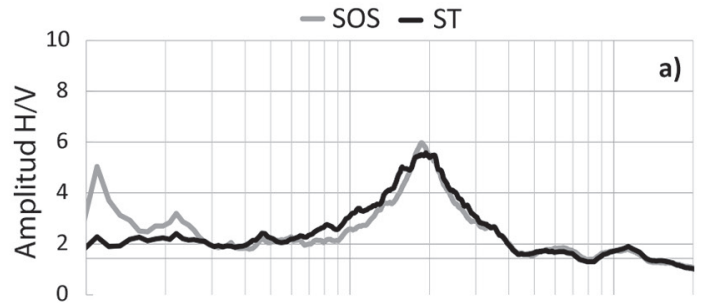

APMR

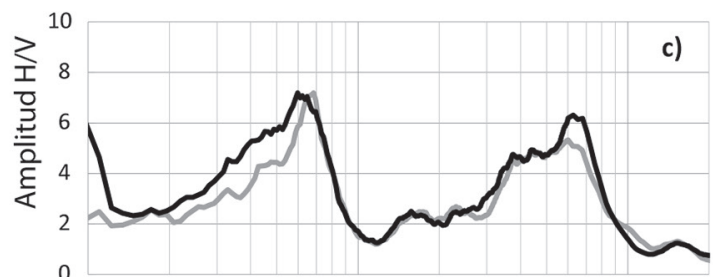

PQUE

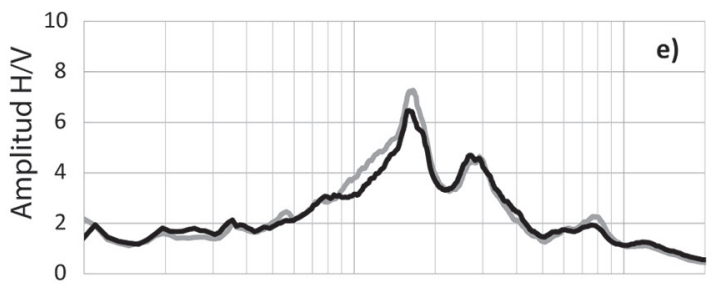

SSBN

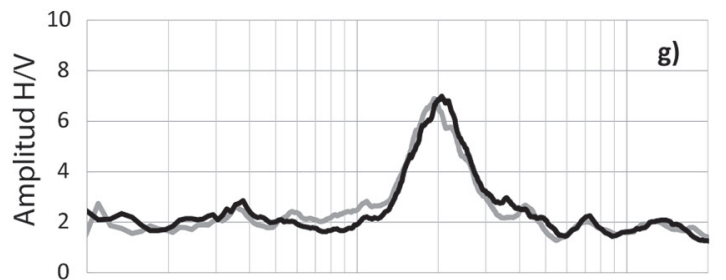

CCRT

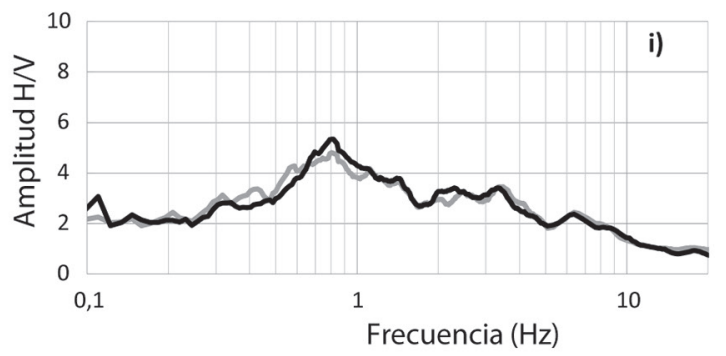

SHTH

- SOS - ST

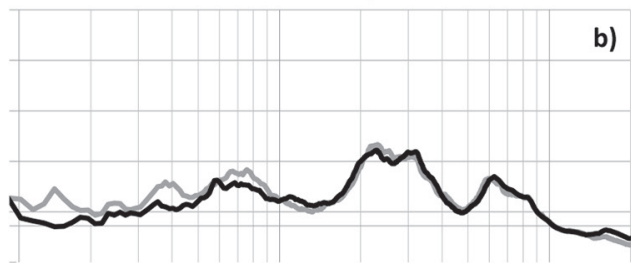

CPAR

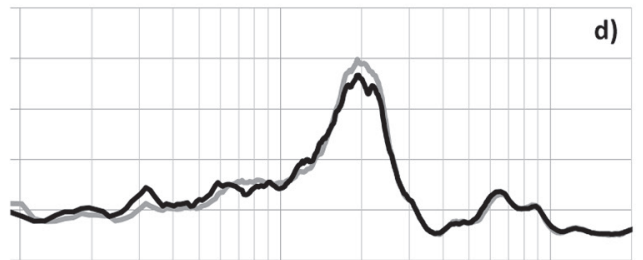

SLPF

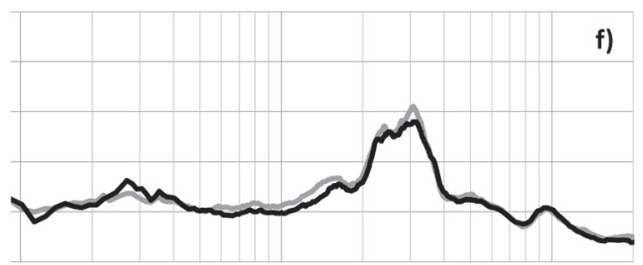

ASRM

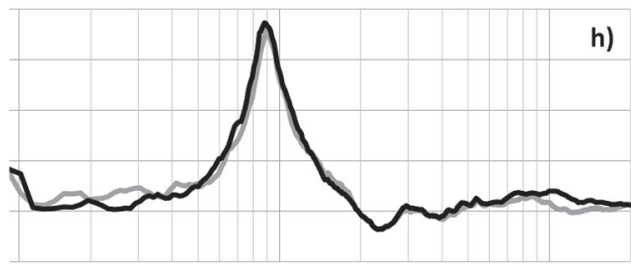

CTEC

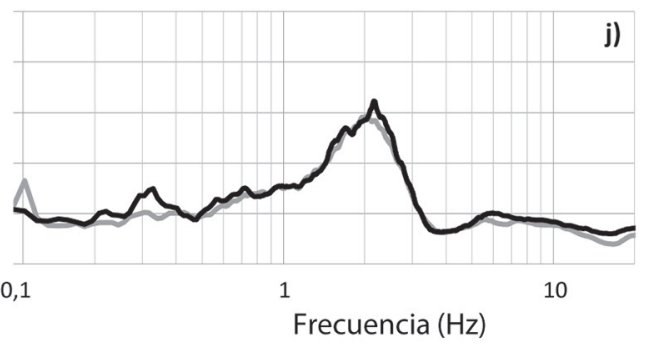

Fig. 10: Comparación entre las técnicas ST (sismo total) y SOS (5 segundos de onda S) en los sitios: AALA, SHTH, APMR, CPAR, PQUE, SLPF, SSBN, ASRM, CCRT y CTEC. 
AFBR
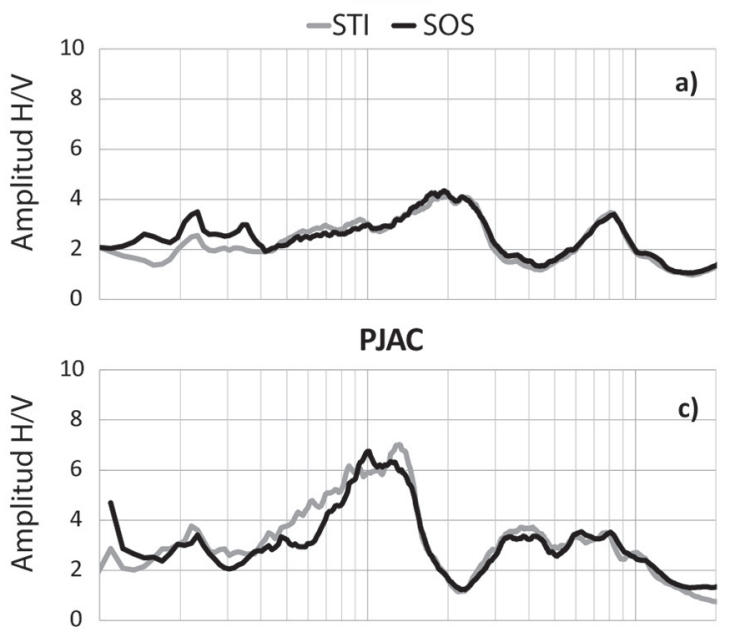

SJUD

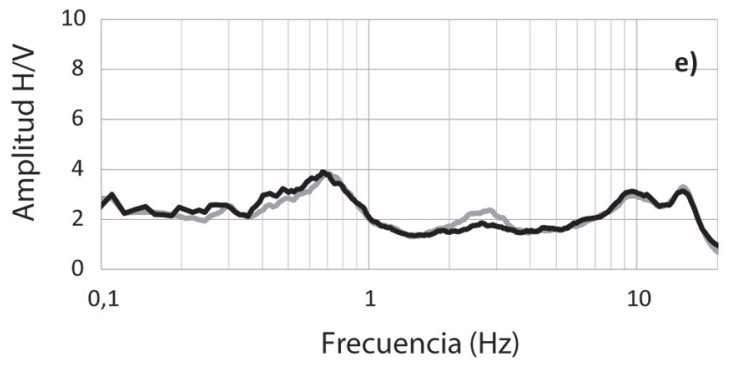

AGRE
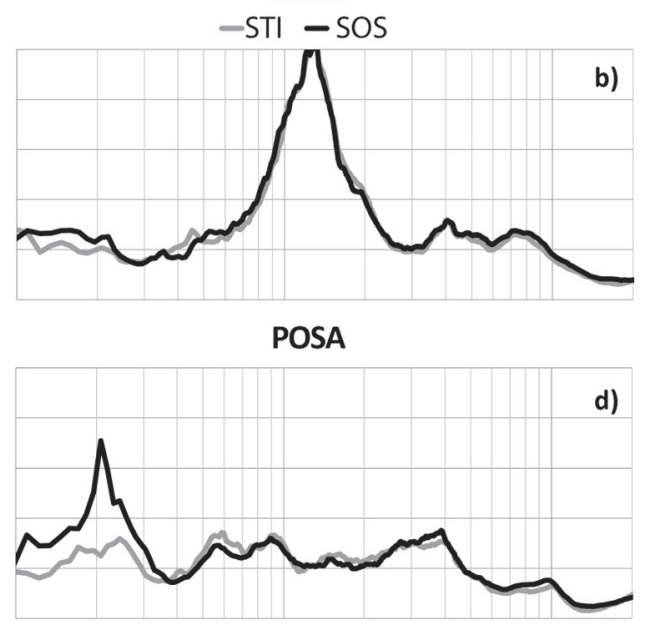

CTBA

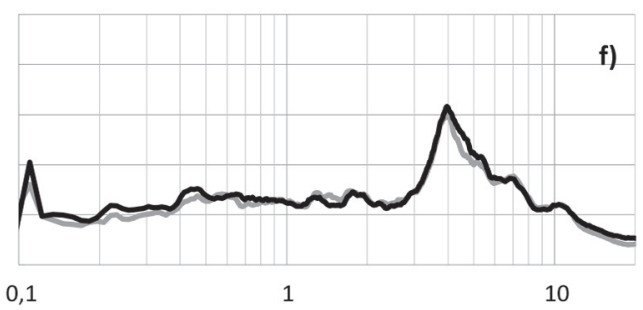

Frecuencia $(\mathrm{Hz})$

Fig. 11: Comparación entre las técnicas ST (sismo total) y SOS (5 segundos de onda S) en los sitios: AFBR, AGRE, PJAC, POSA, SJUD y CTBA.

Al usar sismógrafos pero aplicando las técnicas para vibraciones ambientales (VAS) y sismos (ST), se observa que en la mayoría de los sitios los resultados son semejantes, pero de menor amplitud las correspondientes a VAS, que provienen de ondas con un menor nivel de energía.

La semejanza entre los resultados de la mayoría de las comparaciones entre VAS y ST permite concluir acerca de la confiabilidad para aplicar VAS para determinar la frecuencia fundamental del sitio, a un costo y tiempo mucho menor que ST, ya que la primera técnica se obtiene a partir de vibraciones ambientales con ventanas de 15 minutos aproximadamente, que pueden ser registradas en cualquier momento, mientras que para
ST se requiere contar con varios registros de sismos, lo que implica que para su aplicación deba esperarse meses o años.

Finalmente, al comparar ST con SOS se observan resultados muy semejantes, siendo mucho más sencilla de aplicar la ST, ya que no requiere el trabajo adicional de aplicar algún algoritmo que identifique los distintos arribos de onda y en particular la S.

\section{AGRADECIMIENTOS}

Un agradecimiento a todo el personal del Laboratorio de Ingeniería Sísmica, en especial 
al técnico Esteban Cordero y al asistente Juan Carlos Fonseca por el apoyo en el trabajo de campo y su disposición para ayudar en todo momento y en cualquier labor encomendada.

\section{REFERENCIAS}

BARD, P. Y., 1999: Microtremor measurements: A tool for site effect estimation?.- Stateof-the-art paper, Second International Symposium on the Effects of Surface Geology on seismic motion, Yokohama, Japón, 1998.- En: IRIKURA, K., KUDO, K., OKADA, H. \& SASATAMI, T. (eds): Effects of Surface Geology on Seismic Motion.- Ed. Balkema, Rotterdam: 1251-1279.

DUVAL, A., BARD, P. Y., LEBRUN, B., LACHET, C., RIEPL, J. \& HATZFELD, D., 2001: H/V technique for site response analysis, synthesis of data from various surveys.- Bollettino di Geofisica Teorica ed Applicata, 42(3-4): 267-280.

KONNO, K. \& OHMACHI, T., 1998: Groundmotion characteristics estimated from spectral ratio between horizontal and vertical components of microtremor.- Bull. Seismol. Soc. Amer. 88: 228-241.

KRAMER, S. L., 1996: Geotechnical earthquake engineering.- 653 págs. Ed. Prentice-Hall, New Jersey.

KUDO, K., 1995: Practical estimates of site response, State-of-the- art report.- Proc. of the fifth Int. Conference on Seismic Zonation, Nice, France, 3: 1878-1907.

LACHET, C., \& BARD, P. Y., 1994: Numerical and theoretical investigations on the possibilities and limitations of Nakamura's technique.- J. Phys. of the Earth, 42(5): 377-397.
MUCCIARELLI, M., 1998: Reliability and applicability of Nakamura's technique using microtremors: an experimental approach.J. Earthquake Eng. 2(4): 1-14.

NAKAMURA, Y., 1989: A method of dynamic characteristics estimation of subsurface using microtremor on the ground surface.Railways Technol. Res. Inst. Quaterly Reports, 30(1): 25-33.

NOGOSHI, M. \& IGARASHI, T., (1971): On the amplitude characteristics of microtremor (Part 2) (in Japanese with English abstract).- J. Seismol. Soc. Japan, 24: 26-40.

RIEPL, J., BARD, P. Y., HATZFELD, D., PAPAIOANNOU, C. \& NECHSTEIN, S., 1998: Detailed evaluation of site response estimation methods across and along the sedimentary valley of Volvi (EURO-SEISTEST).- Bull. Seismol. Soc. Amer. 88: 488-502.

ROJAS, V., 2013: Relación entre los procesos volcano-sedimentarios y el neotectonismo de la cuenca lacustrina de Palmares y San Ramón, Costa Rica.- 123 págs. Univ. de Costa Rica, San José [Tesis Lic.].

SCHMIDT, V., 2014: Clasificación de suelos de 15 estaciones acelerográficas mediante el uso de métodos basados en vibraciones ambientales y del parámetro Vs30.- Rev. Geol. Amer. Central, 51: 33-67.

SCHNABEL, P., LYSMER, B. \& SEED, H. B., 1972: SHAKE: A computer program for earthquake response analysis of horizontally layered sites, Report $\mathrm{N}^{\circ} \mathrm{UCB} /$ EERC 72/12.- 102 págs. Earthquake Eng. Res. Center, University of California, Berkeley.

SESAME EUROPEAN RESEARCH PROJECT, 2004: Guidelines for the implementation 
of the $\mathrm{H} / \mathrm{V}$ spectral ratio technique on ambient vibration. Measurements, processing and interpretations.- 62 págs.
European Commission - Research General Directorate, Project No. EVG1CT-2000-00026 SESAME. 
